\title{
Statistical Mechanics of liquids and fluids in curved space
}

\author{
Gilles Tarjus ${ }^{1}$, François Sausset ${ }^{2}$, and Pascal Viot ${ }^{1}$ \\ ${ }^{1}$ Laboratoire de Physique Théorique de la Matière Condensée, \\ Université Pierre et Marie Curie-Paris 6, \\ UMR CNRS 7600, 4 place Jussieu, \\ 75252 Paris Cedex 05, France \\ and \\ ${ }^{2}$ Department of Physics, Technion Haifa, 32000 Israel
}




\section{CONTENTS}

I. Introduction 3

II. Theoretical motivations and physical realizations 4

III. Thermodynamics and boundary effects 6

A. Thermodynamic limit and boundary conditions 6

B. Spherical geometry

C. Hyperbolic geometry 9

IV. Liquid state theory in spaces of constant nonzero curvature 11

A. Statistical mechanics 11

B. Thermodynamic pressure and equation of state 13

C. Correlation functions and integral equations 16

D. Interaction potentials

E. Computer simulations 22

F. Self-motion and diffusion equation 24

V. Curvature effect on the thermodynamics and structure of simple fluids 27

A. Short-range interaction potentials

B. Coulombic systems 32

C. Liquid-gas critical behavior 38

VI. Freezing, jamming and the glass transition 42

A. Geometric frustration 42

B. Glassforming liquid on the hyperbolic plane 46

VII. Ground-state properties, order and defects

VIII. Conclusion 55

A. A recap on Riemannian manifolds 5

B. Periodic boundary conditions on the hyperbolic plane 59 


\section{INTRODUCTION}

The statistical mechanics of liquids and fluids is by now a mature field which has evolved from the study of simple liquids to that of increasingly complex systems and phenomena (see e.g [1]). A research direction which has received only limited attention so far is that of liquids embedded in curved spaces. Although the topic might appear at first sight as a purely theoretical curiosity or only relevant to general relativity, it arises in situations in which particles are adsorbed or confined on a substrate with nonzero curvature, be it the wall of a porous material, the surface of a large solid particle or an interface in an oil-water emulsion. In the latter cases, the fluid is confined to a curved manifold which is two-dimensional. Statistical-mechanical systems in three-dimensional curved spaces and manifolds are not encountered in our everyday life experience, but they are useful as templates or models to study properties whose direct investigation in Euclidean three-dimensional space remains difficult or inconclusive: curvature and geometry then provide additional control parameters to envisage the behavior of a system.

In this article, we review the progress made on the statistical mechanics of liquids and fluids embedded in curved space. Our main focus will be on two-dimensional manifolds of constant nonzero curvature and on the influence of the latter on the phase behavior, thermodynamics and structure of simple liquids. Reference will also be made to existing work on three-dimensional curved space and two-dimensional manifolds with varying curvature. On the other hand, we exclude from the scope of the article substrates with fluctuating geometry, such as membranes[2, 3]. The geometry will always be considered as frozen, providing the background whose metrical and topological characteristics affect the behavior of the embedded fluids, but with no feed-back influence from the latter.

The rest of the paper is organized as follows: in Sec. II] we review physical examples where the curvature of substrate modify the physical properties of systems in comparison with those observed in in Euclidean space. In Sec. III, we discuss the specificities of thermodynamics for liquids coming from the finiteness of space (constant positive curvature) or strong influence of the boundary effects (constant negative curvature). We introduce in Sec. IV elements of liquid state theory for describing the structure and the thermodynamic properties of fluids 
in curves spaces. Section $\mathrm{V}$ is devoted to the influence of the curvature on the structure of liquids as well as the modifications of the Coulomb interaction We consider in Sec. VI illordered dense phases where the geometric frustration prevents the appearance of an ordering transition and slows down drastically the relaxation dynamics of liquids driving the system to a glassy behavior. In Sec. VII, the low-temperature regions of phase diagram is analyzed by means the elastic theory of defects, which reveals the structure of topological defects generally in excess in curved spaces. Concluding remarks are drawn in Sec. VIII.

\section{THEORETICAL MOTIVATIONS AND PHYSICAL REALIZATIONS}

In this section we expand a little more on the reasons for studying fluids in curved spaces. Before moving on to more solid grounds, let us first acknowledge that non-Euclidean geometries are fun and fascinating. Musing about the differences between spherical and hyperbolic worlds, i.e. behavior in spaces of constant positive and negative curvature respectively, is an exciting intellectual experience! At a fundamental level, a nonzero curvature introduces (at least) one extrinsic lengthscale (a "radius of curvature") in the behavior of systems embedded in such curved spaces. One then expects that the "long-distance" properties of the system, namely those involving lengths much larger than the radius (or radii) of curvature, are modified whereas "local" ones should be rather insensitive to curvature in general. Curving space may also change the topology of the substrate, going for instance from an infinite flat plane to a sphere or a torus, which also affects some properties of the embedded system such as the nature of its ordered condensed phases.

From a theoretical point of view, curving space provides an additional control parameter (the Gaussian curvature or the associated radius of curvature for a homogeneous space of constant curvature) for studying the properties of a fluid or a liquid, in addition to the common thermodynamic parameters. This may prove interesting in several situations. First, there are cases for which in the standard Euclidean space, boundary conditions matter. This is true for instance for Coulombic systems in which charged particles interact through a longrange Coulomb potential or in the vicinity of a gas-liquid critical point where correlations extend over the whole system size. The long-range character of the interactions or of the correlations entail the use of boundary conditions, usually periodic boundary conditions, in standard statistical-mechanical treatments. An alternative is provided by the so-called 
"spherical and hyperspherical boundary conditions" [4] 6 , which amount to considering the system on the surface of a sphere (for a two-dimensional space) or a hypersphere (for a threedimensional space). A (hyper)sphere is a homogeneous and isotropic manifold of constant positive Gaussian curvature. It is of finite extent so that no boundary conditions need to be specified. The Euclidean space is then recovered by letting the radius of the (hyper)sphere go to infinity.

Another domain in which curving space proves to be of major theoretical importance is what goes under the concept of "geometric frustration" [7]. The latter describes an incompatibility between the preferred local order in a system and the tiling of the whole space. Geometric frustration has emerged in the theoretical description of glasses and amorphous solids from the consideration of local icosahedral order in metallic glasses. Icosahedral, or more generally poly-tetrahedral order, is favored for local arrangement of atoms [8] but cannot extend to form a periodic tiling of three-dimensional Euclidean space [7, 9]. It has first been realized by Kléman and Sadoc [10], and further developed by several groups [7, 9], that perfect tetrahedral/icosahedral order can exist on the surface of a hypersphere with an appropriately chosen radius. More generally, curving space can be seen as a way to reduce (as in the above example) or increase the amount of geometric frustration in a liquid. Increasing frustration is helpful for liquids of spherical particles in two dimensions. Indeed, the ordinary "flat" space then leads to no frustration. The locally preferred hexagonal order can tile space to produce a triangular lattice. As a result, one-component atomic liquids on a Euclidean plane rapidly order under cooling (or compressing) and do not form glasses. To study glass formation and amorphous packings in such systems, one must introduce curvature. As suggested by Nelson and coworkers [9, 11, 12], one could then mimic frustrated icosahedral order in ordinary three-dimensional space by considering frustrated hexagonal order in the hyperbolic plane, which is a homogeneous and isotropic two-dimensional manifold of constant negative Gaussian curvature.

Up to now, we have focused on purely theoretical motivations for studying curved spaces. As mentioned in the introduction, there are also physical realizations of fluids on twodimensional curved substrates. Such situations occur in adsorption and coating phenomena. Generally, the adsorbing solid substrate is not flat (it could be locally cylindrical or spherical) and an equilibrium fluid monolayer can form on its surface if the fluid-solid attraction is strong enough. There are cases where the curvature can even be quite strong, with the asso- 
ciated length only moderately larger than that of the adsorbate. This happens for instance in the adsorption of gas molecules inside the cavities of zeolite molecular sieves or that of colloidal particles on larger spherical particles in the phenomenon of heterofloculation[13].

Fluid and condensed-phase behavior is also observed at the oil-water interface in emulsions 14 , 15]. The presence of colloidal particles at the interfaces actually stabilize the emulsions, then often referred to as Pickering emulsions[16]. The colloidal particles are irreversibly adsorbed but still mobile at the interface which is usually the surface of a spherical droplet. Up to now, most of the existing experiments focus on dense, crystal-like arrangements of colloidal particles on spherical droplets [14, 15, 17, 20], but one could imagine studying less dense, liquid-like behavior[21]. One could also consider adsorbed colloidal particles on more exotic interfaces such as the infinite periodic minimal surfaces formed for instance by amphiphilic molecules in the presence of water 22 24]. Such surfaces have negative, but varying, curvature and are periodic in all three directions. On the other hand, we do not discuss in this review situations in which the geometry of the substrate changes when coated by colloidal particles, as may occur for bubbles in foams for instance [25, 26]. In addition, we only briefly allude to crystalline-like order on curved substrates, as this important topic has been recently extensively reviewed by Bowick and Giomi[27].

\section{THERMODYNAMICS AND BOUNDARY EFFECTS}

\section{A. Thermodynamic limit and boundary conditions}

Thermodynamics, and more generally all dynamic and structural properties of a macroscopic system, are retrieved with statistical mechanics by taking the thermodynamic limit in which one let the number of particles and the volume of the system go to infinity while keeping the density finite. This also ensures equivalence between the various thermodynamic statistical ensembles. Such a procedure is, at least conceptually, easy to implement in Euclidean space when both interactions and spatial correlations between particles are shortranged. So long as the system is finite, the properties under study depend on the choice of the boundary conditions, e.g. free or periodic, but as one takes the thermodynamic limit the contribution of the boundary vanishes irrespective of the chosen conditions (the rate

of convergence does however depend on the boundary conditions). The same behavior also 
characterizes manifolds of varying curvature that are of infinite extension and can be embedded in Euclidean space. Note however, that in addition to the subtleties occurring for long-range, e.g. Coulombic, interactions or long-range correlations near a critical point, some care must be exerted for anisotropic and multi-connected manifolds as the spatial extent of the system can be made infinite only in certain directions.

Quite different is the behavior of homogeneous spaces of constant nonzero curvature. Spherical geometry associated with a positive curvature indeed leads to spaces of finite extent whereas hyperbolic geometry associated with a negative curvature allows for infinite spatial extent but with a strong effect of the boundary conditions. In what follows, we discuss these two main geometries, specializing for simplicity to the two-dimensional case.

\section{B. Spherical geometry}

In two dimensions, the space of constant positive curvature is the surface $S_{2}$ of a sphere. If $R$ is the radius of the sphere, $1 / R^{2}$ is its Gaussian curvature and its area $4 \pi R^{2}$ is obviously finite. Taking the thermodynamic limit $R \rightarrow \infty$ "flattens" the space and one then recovers the Euclidean plane. The same is true in higher dimensions. If one wishes to consider fluids on spherical substrates of constant curvature $1 / R^{2}$, one must resort to the thermodynamics of finite systems. This topic has been (ands still is) extensively discussed. For small systems, one route is provided by Hill's formulation [28] of the thermodynamics of small systems: one starts with a finite canonical system and consider a special ensemble formed by replicas of this system. The intensive properties of the system depend separately on the number of particles $N$ on a sphere and the total area $A=\pi R^{2}$ of the sphere instead of being functions of the particle density $\rho=N / A$ only. This formalism leads to the introduction of an additional "pressure" on top of the familiar spreading pressure $p$ of the fluid on the curved substrate, which invalidates for small enough systems the Gibbs-Duhem equation[13, 28].

The dependence on the finite size and finite number of particles can be illustrated by looking at the virial coefficients of the density expansion of the spreading pressure. This expansion reads 4 ]

$$
\frac{\beta P}{\rho}=1+\sum_{j=2}^{\infty} B_{j}(N, R) \rho^{j-1},
$$

where $\beta=1 / k_{B} T$ and the first virial coefficients $B_{j}(N, R)$ for a canonical system are given 
by [4]

$$
\begin{aligned}
B_{2}(N, R) & =\left(1-\frac{1}{N}\right)\left(B_{2, M}(R)-\frac{R}{2} B_{2, M}^{\prime}(R)\right) \\
B_{3}(N, R) & =\left(1-\frac{1}{N}\right)\left[\left(1-\frac{2}{N}\right)\left(B_{3, M}(R)-\frac{R}{4} B_{3, M}^{\prime}(R)\right)\right. \\
& \left.+\frac{2}{N} B_{2, M}(R)\left(B_{2, M}(R)-\frac{R}{2} B_{2, M}^{\prime}(R)\right)\right],
\end{aligned}
$$

with $B_{j, M}^{\prime}(R)=\partial B_{j, M}(R) / \partial R$. The $B_{j, M}(R)^{\prime}$ 's are the Mayer cluster integrals which are defined as usual, for a spherically symmetric pair interaction potential $u(r)$ by

$$
B_{2, M}(R)=-\frac{1}{2 A(R)} \int_{A(R)} \int_{A(R)} d S_{1} d S_{2} f_{12}
$$

and

$$
B_{3, M}(R)=-\frac{1}{3 A(R)} \int_{A(R)} \int_{A(R)} \int_{A(R)} d S_{1} d S_{2} d S_{3} f_{12} f_{13} f_{23},
$$

with $f_{i j}=\exp \left(-\beta u\left(r_{i j}\right)\right)-1, r_{i j}$ being the geodesic distance between points $i$ and $j$ on $S_{2}, A(R)=4 \pi R^{2}$, and $d S$ is the differential area on $S_{2}$. The first virial coefficients have been computed for hard disks on $S_{2}$ (and hard spheres on $S_{3}$ ) and the resulting approximate equation of state for the spreading pressure compared to simulation results. This will be discussed later on. Note that we have interpreted above the interaction between particles as acting in curved space and therefore depending on the geodesic distance on $S_{2}$. This corresponds to the "curved line-of-force" case[13]. When the curved space can be embedded in a higher-dimensional Euclidean space (here, the sphere $S_{2}$ in the 3-dimensional Euclidean space $E_{3}$ ), one can also envisage interactions acting through the embedding Euclidean space, which corresponds to the "Euclidean line-of-force" case[13]. Which case arises depends on the physics of the system under consideration. Most existing studies use the "curved lineof-force" description and this is what we shall do throughout this article.

It is worth stressing again that spherical geometry does not allow one to study finitesize effects at both constant curvature and constant particle density. This however may be viewed as a boon if one wishes to study the flat case in the thermodynamic limit. Spherical boundary conditions with spheres of increasing radius indeed offer an alternative to the more common periodic boundary conditions implemented directly in the Euclidean space. 


\section{Hyperbolic geometry}

If finiteness is the characteristic of spherical substrates, a quite different and singular behavior takes place in hyperbolic world. The hyperbolic plane $H_{2}$, which is a homogeneous, simply connected two-dimensional manifold of constant negative curvature, is of infinite extent [29, 30], (see Appendix A). The thermodynamic limit, at constant curvature and constant particle density, can therefore be taken in such a space. However, a peculiar feature of the hyperbolic geometry is that because of the exponential character of the metric at large distance, the boundary of a finite region of $\mathrm{H}_{2}$ grows as fast as the total area of this region when the size of the latter increases. To be more specific, consider the hyperbolic plane of Gaussian curvature $K=-\kappa^{2}$. The metric in polar coordinates $(r, \phi)$ is given by

$$
d s^{2}=d r^{2}+\left(\frac{\sinh (\kappa r)}{\kappa}\right)^{2} d \phi^{2} .
$$

This form makes apparent the connection with the spherical metric of the sphere $S_{2}$ which is obtained by replacing in the above expression $\kappa$ by $i R^{-1}$. See Appendix A. We shall refer to $\kappa^{-1}$ as the "radius of curvature" of the hyperbolic plane. Still in polar coordinates, the differential area is expressed as

$$
d S=\frac{\sinh (\kappa r)}{\kappa} d r d \phi
$$

which again is the counterpart of the expression for $S_{2}$ with the replacement of $\kappa$ by $i R^{-1}$. It is a simple exercise in hyperbolic geometry to show that the area of a disk of radius $r$ is given by $A(r)=2 \pi \kappa^{-2}(\cosh (\kappa r)-1)$ whereas its perimeter is equal to $P(r)=2 \pi \kappa^{-1} \sinh (\kappa r)$ (see Appendix A). For a large radius such that $\kappa r \gg 1$, one then finds as announced above that both $A(r)$ and $P(r)$ grow exponentially with the distance $r$, as $\exp (\kappa r)$.

As a result of the above property, the boundary effects are never negligible, even in the thermodynamic limit! An illustration is provided by the ideal gas behavior 31, 32]. The canonical partition $Q_{1}^{i d}$ can be obtained from the eigenenergies of the Schrödinger equation for a single particle in a large domain $\Sigma$ of $H_{2}$. This requires determining the spectrum of the Laplace-Beltrami operator with specified boundary conditions. For an open domain $\Sigma$, the result, obtained as an expansion in the inverse of the de Broglie thermal wavelength $\lambda_{T}=\sqrt{2 \pi \hbar^{2} /\left(m k_{B} T\right)}$ is as follows 3133].

$$
Q_{1}^{i d}(T)=\frac{A(\Sigma)}{\lambda_{T}^{2}} \pm \frac{P(\Sigma)}{4 \lambda_{T}}+\frac{1}{12 \pi}\left[\int_{\Sigma} d S K+\int_{\partial \Sigma} d s K_{g}\right]+O\left(\lambda_{T}\right),
$$


where $K=-\kappa^{2}$ is the Gaussian curvature, $K_{g}$ the geodesic curvature of the boundary $\partial \Sigma$, and the plus or minus signs of the second term correspond to Neumann or Dirichlet boundary conditions for the wavefunction on $\partial \Sigma$. The perimeter $P(\Sigma)$ being of the same order as the area $A(\Sigma)$ for a large domain, the above expression proves the dependence of the ideal gas partition function on the boundary conditions in the thermodynamic limit.

If one nonetheless insists on defining "bulk" thermodynamic properties in $H_{2}$, a solution is to consider periodic boundary conditions. The procedure however is quite complex in $\mathrm{H}_{2}$ as one must change the boundary condition when increasing the area of the primitive cell in which the fluid is embedded. This is detailed in Ref.[34] and is briefly explained in Appendix B. Imposing periodic boundary conditions amounts to replicating a primitive cell (chosen as a regular polygon) to realize a tiling of the whole hyperbolic plane. From a topological point of view, this procedure leads to a compact "quotient space" which is obtained by identifying in a specific manner the edges of the primitive cell by pairs. In the Euclidean case, it is well known that one obtains in this way for square and hexagonal periodic boundary conditions a one-hole torus. For the hyperbolic case, tori of genus $g$ (i.e. with $g$ holes) with $g \geq 2$ are generated [34]. Under such periodic boundary conditions, the boundary terms associated with $\partial \Sigma$ (and $P(\Sigma)$ ) disappear as the manifold is now compact (boundaryless) and one is left with

$$
Q_{1}^{i d}(T)=A(\Sigma)\left[\frac{1}{\lambda_{T}^{2}}-\frac{\kappa^{2}}{12 \pi}\right],
$$

which is positive and proportional to the area of the system if the de Broglie wavelength is small enough compared to the radius of curvature $\kappa^{-1}$. Note that due to the Gauss-Bonnet theorem[7], the area $A$ of a primitive cell associated with a compact quotient space of genus $g$ is fixed and equal to

$$
A=2 \pi \kappa^{-2}(g-1),
$$

where, we recall, $g \geq 2$ on $H_{2}$.

This, rather long, detour via the ideal-gas limit illustrates that (i) boundary effects always have to be considered in hyperbolic geometry, even in the thermodynamic limit, and (ii) "bulk" thermodynamic quantities can be defined by using periodic boundary conditions. Actually, a poor man's way of studying such bulk properties is to follow a procedure commonly employed in analytical studies of statistical-mechanical systems defined on a so-called "Bethe lattice" [35, 36]. This amounts to focusing on the "deep interior" of a very large do- 
main, far enough from the boundary and restricting in all spatial integrals that appear in the calculation of the fluid properties to this deep interior. In practice, one should exclude from computations a boundary region, which is taken as a region of width (or width whose ratio to the linear size of the system goes to zero when the latter goes to infinity (near the boundary of an otherwise very large domain), and subsequently take the thermodynamic limit. We shall come back to this point in the following sections.

\section{LIQUID STATE THEORY IN SPACES OF CONSTANT NONZERO CURVA- TURE}

\section{A. Statistical mechanics}

We consider a liquid at equilibrium in canonical conditions (fixed temperature $T$, volume $V$ and number of particles $N$ ) in a $d$-dimensional Riemannian manifold. The latter is equipped with a metric that can be expressed in a $d$-dimensional set of coordinates $\mathbf{x}=$ $\left(x_{1}, . ., x_{d}\right)$ as

$$
d s^{2}=\sum_{i, j=1}^{d} g_{i j}(\mathbf{x}) d x^{i} d x^{j}
$$

where $g_{i j}(\mathbf{x})$ is the metric tensor, from which one can also derive by standard differential geometry the geodesic equation, the Levi-Civita connection and Riemann (curvature) tensor [7, 37, 38]. Thee infinitesimal element of volume then reads

$$
d S=\sqrt{|g(\mathbf{x})|} \prod_{i=1}^{d} d x_{i}
$$

where $g(\mathbf{x})$ is the determinant of the metric tensor. The geometry is assumed to be frozen, i.e. $g_{i j}(\mathbf{x})$ is fixed in each point of the manifold and is not influenced by the behavior of the liquid. For simplicity, we restrict the presentation to a one-component atomic liquid with pairwise additive interactions $u(r)$ that only depend on the geodesic distance between atoms

computed with the metric in Eq. (4.1) ("curved line of force", see Sec. III). The canonical partition function is given by

$$
Q_{N}(V, T)=Q_{N}^{i d}(V, T) \frac{Z_{N}(V, T)}{V^{N}}
$$


where $Q_{N}^{i d}(V, T)=\frac{Q_{1}^{i d}(V, T)^{N}}{N !}$, is the ideal-gas contribution already considered in the previous section and $Z_{N}(V, T)$ is the configurational integral defined as

$$
Z_{N}(V, T)=\int_{V} \ldots \int_{V} d S_{1} \ldots d S_{N} \exp \left[-\frac{\beta}{2} \sum_{i, j=1}^{N} u\left(r_{i j}\right)\right]
$$

with $d S_{i}$ given by Eq. 4.2 and the prime on the double sum indicating that the term $i=j$ is excluded. As was discussed in some detail in Sec. III, a rigorous definition of the thermodynamic quantities may be subtle in curved space due either to the finiteness of the system (spherical geometry) of to the importance of the boundary conditions (hyperbolic geometry). In addition to this fundamental issue, there is a practical difficulty when considering spaces which are not homogeneous and isotropic, for instance manifolds with spatially varying curvature. Thermodynamics may then still be defined but the intrinsic inhomogeneity or anisotropy of the embedding space makes practical calculations very complex: for instance, the harmonic analysis (Fourier analysis, convolution theorem, Laplace eigenvalues, etc) is only defined on symmetric spaces, namely manifolds of constant curvature. As far as we know, there are virtually no existing statistical-mechanical studies of this kind. Therefore, in what follows, and with the goal to illustrate the influence of the substrate curvature on the behavior of the fluid, we only consider homogeneous manifolds of constant curvature.

From the canonical partition function, one has access to the free-energy, the energy and the thermodynamic pressure. The latter is defined as

$$
\beta P=\left(\frac{\partial \ln \left(Q_{N}\right)}{\partial V}\right)_{T, N} .
$$

As noted in Sec. III, the above expression corresponds to the "spreading pressure" for a spherical substrate. In the case of a hyperbolic geometry, we will focus on the "bulk thermodynamic pressure" which is defined either through the use of periodic boundary conditions or by restricting the configurational integrals to the deep interior of the system, far enough from the boundary (see however the discussion in Sec. VB). In the canonical ensemble, one can also define as usual the $n$-particle correlation (or distribution) functions through

$$
g_{N}^{(n)}\left(\mathbf{r}_{1}, \mathbf{r}_{2}, \ldots, \mathbf{r}_{n}\right)=\frac{V^{N}}{Z_{N}} \int_{V} \ldots \int_{V} d S_{n+1} \ldots d S_{N} \exp \left[-\frac{\beta}{2} \sum_{i, j=1}^{N} u\left(r_{i j}\right)\right]
$$

The above expression is valid in the spherical geometry for a large enough number $N$ of particles (otherwise, there are $1 / N$ corrections: see Refs [4, 13]) and in the hyperbolic geometry 
for the "bulk" functions (see above). In both cases, the embedding space can be considered as homogeneous and isotropic so that $g_{N}^{(1)}\left(\mathbf{r}_{1}\right)=1, g_{N}^{(2)}\left(\mathbf{r}_{1}, \mathbf{r}_{2}\right)$ only depends on the geodesic distance $r_{12}$, etc.

Having defined the thermodynamic quantities and the correlation functions for fluids constrained in curved spaces (of constant curvature), one can derive the various relations among them. These relations take simple forms in the case where the interactions are pairwise additive (as considered here) and then only involve the pair correlation function.

\section{B. Thermodynamic pressure and equation of state}

Whereas the expression for the excess internal energy in terms of the pair correlation function is a straightforward extension of the standard formula in Euclidean space[39], that for the thermodynamic pressure requires more caution in its derivation. We treat the case of positive curvature (spherical geometry) and negative curvature (hyperbolic geometry) separately. Indeed, in the former situation, a change of the total volume, as required from the thermodynamic definition in Eq. 4.5), implies a change of curvature while the total volume can be changed at constant curvature in the latter case. For ease of exposition, we deal with two-dimensional manifolds, but the reasoning is easily extended to higher dimensions.

Let us consider first a spherical substrate formed by the surface $S_{2}$ of a sphere of radius $R$. The spherical metric can be conveniently expressed in polar coordinates with two angles $\theta$ (colatitude) and $\phi$ (longitude) as

$$
d s^{2}=R^{2} \sin (\theta) d \theta d \phi
$$

or equivalently, by introducing the geodesic distance to the north pole $r=R \theta(0 \leq r \leq \pi R)$, as $d s^{2}=\left[R \sin \left(\frac{r}{R}\right)\right] d r d \phi$, which makes the comparison with the Euclidean and hyperbolic cases more direct. The configurational integral in Eq. 4.4 can be reexpressed as

$$
Z_{N}(A, T)=R^{2 N} \int_{0}^{\pi} d \theta_{1} \sin \left(\theta_{1}\right) \int_{0}^{2 \pi} d \phi_{1} \ldots \int_{0}^{\pi} d \theta_{N} \sin \left(\theta_{N}\right) \int_{0}^{2 \pi} d \phi_{N} \exp \left[-\frac{\beta}{2} \sum_{i, j=1}^{N} u\left(R \theta_{i j}\right)\right]
$$

where $\theta_{i j}=\theta_{i}-\theta_{j}$ and the total area $A=4 \pi R^{2}$. By using Eq. (4.3) and Eq. 4.5 with the 
derivative with respect to the "volume" replaced by $\partial A=2\left(\frac{A}{R}\right) \partial R$, one finds

$$
\beta P=\left.\frac{R}{2 A Z_{N}} \frac{\partial}{\partial R} Z_{N}\right|_{N, T},
$$

which, after inserting Eq. 4.8) and the definition of the pair correlation function in Eq. 4.6, gives

$$
\begin{gathered}
\beta P=\frac{N}{A}-\frac{\beta R}{4 A} \sum_{i, j=1}^{N} \frac{R^{4}}{A^{2}} \int_{0}^{\pi} d \theta_{i} \sin \left(\theta_{i}\right) \int_{0}^{2 \pi} d \phi_{i} \int_{0}^{\pi} d \theta_{j} \sin \left(\theta_{j}\right) \\
\int_{0}^{2 \pi} d \phi_{j} u^{\prime}\left(R \theta_{i j}\right) \theta_{i j} g_{N}^{(2)}\left(R \theta_{i}, \phi_{i}, R \theta_{j}, \phi_{j}\right),
\end{gathered}
$$

where $u^{\prime}(r)=\frac{d}{d r} u(r)$. Finally, by using the homogeneity and isotropy of space, introducing the particle density $\rho=N / A$, and considering $N \gg 1$, one arrives at the following expression for the equation of state [4, 13]

$$
\frac{\beta P}{\rho}=1-\frac{\beta \pi \rho R}{2} \int_{0}^{\pi R} d r r \sin \left(\frac{r}{R}\right) u^{\prime}(r) g(r),
$$

where $g(r) \equiv g_{N}^{(2)}(r)$ is the radial distribution function depending on the geodesic distance $r$ between pairs of particles. The expression can be generalized to small $N$ systems and to high dimensions as well[4, 40,42].

We now move on to the hyperbolic plane $H_{2}$. The total area can be varied at constant (negative) curvature $K=-\kappa^{2}$, but to avoid boundary problems (see Sec. III) in computing the bulk thermodynamic pressure, we use a variant of the Green-Bogoliubov method[43, 44]. The derivative in Eq. 4.5 is performed via an affine transformation of the elementary area element,

$$
d S^{\prime}=(1+\xi) d S
$$

with $\xi$ an infinitesimal parameter. Due to the form of the hyperbolic metric (see Sec. III and Appendix A), this transformation leaves the polar angle $\phi$ unchanged, whereas the radial coordinate $r$ becomes

$$
r^{\prime}=r \xi \frac{(\cosh (\kappa r)-1)}{\kappa \sinh (\kappa r)}+O\left(\xi^{2}\right)
$$

which, contrary to the Euclidean case, amounts to a nonlinear transformation of the coordinate. We then consider the infinitesimal variation of the configurational integral which is generated by the above transformation. Taking advantage of the homogeneity and isotropy 
of space (in the bulk), one finds

$$
\delta \ln \left(Z_{N}(A, T)\right)=N \xi-\frac{\beta \rho^{2}}{2} A \int d S g(r) \delta u(r)
$$

where $d S=\kappa^{-1} \sinh (\kappa r) d r d \phi$ and $\delta u(r)$ is the infinitesimal change of the pair potential which, by using Eq. 4.13), can be written as

$$
\delta u(r)=\xi \frac{(\cosh (\kappa r)-1)}{\kappa \sinh (\kappa r)} u^{\prime}(r)+O\left(\xi^{2}\right) .
$$

The final result reads 44

$$
\frac{\beta P}{\rho}=1-\frac{\beta \pi \rho}{2 \kappa^{2}} \int_{0}^{\infty} d r(\cosh (\kappa r)-1) u^{\prime}(r) g(r),
$$

where we have taken the thermodynamic limit by letting the range of integration go to infinity. The above expression is the equation of state for the bulk thermodynamic pressure in $\mathrm{H}_{2}$. In the specific case of Coulombic systems, other types of pressures can be defined [45]: this will be discussed in section $\mathrm{VB}$.

Note that when the curvature goes to zero, i.e. when $R \rightarrow \infty$ in Eq. (4.11) and when $\kappa \rightarrow 0$ in Eq. (4.16), both the spherical and hyperbolic equations of state reduce to the Euclidean one,

$$
\frac{\beta P}{\rho}=1-\frac{\beta \pi \rho}{4} \int_{0}^{\infty} d r r^{2} u^{\prime}(r) g(r) .
$$

However, the results on $H_{2}$ and $S_{2}$ are not simply related by replacing $\kappa$ by $i R^{-1}$ (as it is true for the metric, see Sec. III and Appendix A).

To illustrate the influence of curvature, one may consider the virial expansion of the equation of state, which describes the low-density fluid: see Eq. (3.1). The virial coefficients can be derived by a direct expansion of the partition function or by inserting the density expansion of the radial distribution function in the equation of state, Eqs. 4.11) or 4.16. The first correction to ideal-gas behavior is obtained by setting $g(r)=e^{-\beta u(r)}$, which leads after integrating by parts to

$$
\left.B_{2}\right|_{S_{2}}=-\frac{\pi R}{2} \int_{0}^{\pi R} d r\left(\sin \left(\frac{r}{R}\right)+\frac{r}{R} \cos \left(\frac{r}{R}\right)\right)\left(e^{-\beta u(r)}-1\right)
$$

and

$$
\left.B_{2}\right|_{H_{2}}=-\frac{\pi}{\kappa} \int_{0}^{\infty} d r \sinh (\kappa r)\left(e^{-\beta u(r)}-1\right)
$$


One checks that both expressions reduce to the Euclidean formula for the second virial coefficient when the curvature goes to zero. By introducing as in Sec. III the usual Mayer integral with the appropriate metric,

$$
B_{2, M}\left(R \text { or } \kappa^{-1}\right)=-\frac{1}{2} \int d S f(r)
$$

with $f(r)=e^{-\beta u(r)}-1$, it is easy to check that $\left.B_{2}\right|_{H_{2}}$ is equal to the corresponding Mayer integral, whereas $\left.B_{2}\right|_{S_{2}}$ verifies Eq. (3.2) (with, here, $N \gg 1$ ). The difference of behavior between $\mathrm{H}_{2}$ and $S_{2}$ reflects the property that the spherical manifold is finite and that its total area can only be varied by changing the curvature, i.e. the radius of the sphere.

\section{Correlation functions and integral equations}

In liquid-state theory, it is well established that density expansions are only of limited use for describing the liquid phase and that a more fruitful approach consists in deriving approximate integral equations for the pair correlation functions [39. This is based on the Ornstein-Zernike equation that relates the radial distribution function $g(r)$, or more precisely the so-called total pair correlation function $h(r)=g(r)-1$, to the direct correlation function $c(r)$ :

$$
h(r)=c(r)+\rho \int_{\Sigma} d S^{\prime} h\left(r^{\prime}\right) c\left(t\left(\mathbf{r}, \mathbf{r}^{\prime}\right)\right),
$$

where $\Sigma$ is either $S_{2}$ and $H_{2}$ (again, the formalism is easily generalized to higher-dimensional non-Euclidean Riemannian manifolds of constant curvature) and where $t\left(\mathbf{r}, \mathbf{r}^{\prime}\right)$ is the modulus of the (geodesic) displacement associated with an element of the spherical or hyperbolic translation group. Eq. (4.21) should be considered as a "bulk" property in the case of $H_{2}$, with all boundary effects removed when taking the thermodynamic limit. In the case of $S_{2}$, where finiteness is a source of additional difficulties compared to the Euclidean space, we consider large enough systems so that the number of particles $N$ can be taken as a continuous variable and the explicit $1 / N$ corrections can be neglected (see Sec. III). The Ornstein-Zernike equation can then be interpreted as expressing the Legendre transform between canonical and grand-canonical ensembles, the direct correlation function $c(r)$ be-

ing the second functional derivative of the grand potential with respect to local density fluctuations 39. Again, this interpretation, as well as the equivalence between thermodynamic ensembles, is rigorous in the case of bulk properties in hyperbolic geometry but 
requires some caution in spherical geometry when small systems are investigated[13, 28]. With this proviso in mind, one can derive the compressibility relation,

$$
\frac{\rho \chi_{T}}{\beta}=1+\rho \int_{\Sigma} d S h(r)=\frac{1}{1-\rho \int_{\Sigma} d S c(r)},
$$

where $\chi_{T}$ is the isothermal compressibility and the second relation is a consequence of the Ornstein-Zernike equation.

For homogeneous spaces of constant curvature, it is convenient to reexpress the OrnsteinZernike equation by using a generalization of the Fourier transform. Harmonic analysis on such non-Euclidean spaces has been developed and, in a nutshell, the plane waves used in conventional Fourier transform for a Euclidean space are replaced by the eigenfunctions of the appropriate Laplace-Beltrami operator in curved space (see Appendix A). On the sphere $S_{2}$, one makes use of the Fourier expansion in terms of spherical harmonics expressed with the two angles $\theta$ (colatitude) and $\phi$ (longitude). Any function $f(\theta, \phi)$ (recall that $r=R \theta$ ) can be written as

$$
f(\theta, \phi)=\sum_{k=0}^{\infty} \sum_{|l| \leq k} \hat{f}(k, l) Y_{k l}(\theta, \phi)
$$

where

$$
\hat{f}(k, l)=\int_{0}^{\pi} d \theta \sin (\theta) \int_{0}^{2 \pi} d \phi f(\theta, \phi) Y_{k l}^{*}(\theta, \phi)
$$

and a star denotes the complex conjugate. For a function which is independent of $\phi$ (namely an isotropic function), Eq. (4.23) reduces to a Legendre transform

$$
f(\theta)=\sum_{k=0}^{\infty} \hat{f}_{k} P_{k}(\cos (\theta))
$$

where

$$
\hat{f}_{k}=\left(\frac{2 k+1}{2}\right) \int_{-1}^{1} d x P_{k}(x) f(\arccos (x))
$$

and $P_{k}(x)$ is the $k$ th Legendre polynomial. Convolutions are easily calculated so that the Ornstein-Zernike equation takes the form [46]

$$
1+\rho \frac{4 \pi R^{2}}{(2 k+1)} \hat{h}_{k}=\frac{1}{1-\rho \frac{4 \pi R^{2}}{(2 k+1)} \hat{c}_{k}} .
$$

It is easily checked that $4 \pi R^{2} \hat{h}_{0}=\int_{S_{2}} d S h(r)$, so that the $k=0$ term of Eq. 4.26 provides the compressibility via Eq. 4.22] [46]. 
The counterpart of the Fourier transform on the hyperbolic plane $H_{2}$ is the FourierHelgason transform[37, 47]. For a generic function $f$ of the polar coordinates $(r, \phi)$, it is defined as 37]

$$
\tilde{f}(n, t)=\kappa^{-1} \int_{0}^{\infty} d r \int_{0}^{2 \pi} d \phi \sinh (\kappa r) e^{i n \phi} P_{-1 / 2+i t}^{n}(\cosh (\kappa r)) f(r, \phi),
$$

whereas the inverse Fourier-Helgason transform reads

$$
f(r, \phi)=\frac{\kappa^{2}}{2 \pi} \sum_{n=-\infty}^{+\infty}(-1)^{n} \int_{0}^{\infty} d t t \tanh (\pi t) e^{-i n \phi} P_{-1 / 2+i t}^{-n}(\cosh (\kappa r)) \tilde{f}(n, t) .
$$

For an isotropic function $f(r)$, the Fourier-Helgason function reduces to a Mehler-Fock transform [48]; the dependence on $n$ disappears and, after rewriting $t=\frac{k}{\kappa}$, one has

$$
\tilde{f}(k)=2 \pi \kappa^{-1} \int_{0}^{\infty} d r \sinh (\kappa r) P_{-1 / 2+i k / \kappa}(\cosh (\kappa r)) f(r),
$$

and

$$
f(r)=\frac{1}{4 \pi} \int_{0}^{\infty} d k k \tanh \left(\frac{\pi k}{\kappa}\right) P_{-1 / 2+i k / \kappa}(\cosh (\kappa r)) \tilde{f}(k) .
$$

In the above expressions, $P_{-1 / 2+i t}^{n}(x)$ is a Legendre function of the first kind (conical function) and $P_{-1 / 2+i t}(x)=P_{-1 / 2+i t}^{0}(x)$. The Fourier-Helgason transform satisfies the convolution theorem, so that the Ornstein-Zernike equation, Eq. 4.21, can be rewritten as

$$
1+\rho \tilde{h}_{k}=\frac{1}{1-\rho \tilde{c}_{k}}
$$

Both Eqs. 4.32 and 4.27) converge to the usual Euclidean equation with $\frac{4 \pi R^{2}}{(2 k+1)} \hat{h}_{k}$ and $\tilde{h}(k)$ reducing to the standard Fourier transform when the curvature goes to zero.

One again encounters the peculiarities of curved spaces that have already been pointed out. We have mentioned above the difficulty associated with finiteness in the spherical geometry. A quite different feature occurs in hyperbolic space. One indeed finds that the $k=0$ component of the Fourier-Helgason/Mehler-Fock transform,

$$
\tilde{h}(k=0)=2 \pi \kappa^{-1} \int_{0}^{\infty} d r \sinh (\kappa r) P_{-1 / 2}(\cosh (\kappa r)) f(r),
$$

is different than the integral of $h(r)$ over the whole space, i.e.,

$$
\tilde{h}(k=0) \neq 2 \pi \kappa^{-1} \int_{0}^{\infty} d r \sinh (\kappa r) f(r) .
$$


Actually, $\tilde{h}(k=0)$ is always smaller than the integral. As a result, the $k=0$ component of Eq. (4.32 does not give the compressibility of the fluid. We shall dwell more on this issue when considering the liquid-gas critical point.

The starting point of the integral equation approach to the structure and the thermodynamics of liquids is that the direct correlation function $c(r)$ is simpler and shorter-ranged than $h(r)$. Is is therefore a better candidate for devising approximations. Common approximations that can also be used in homogeneous curved spaces are the Percus-Yevick (PY) and hypernetted chain (HNC) closures, in which

$$
\begin{aligned}
& c(r)=(1+\gamma(r))[\exp (-\beta u(r))-1] \\
& c(r)=\exp (-\beta u(r)+\gamma(r))-(1+\gamma(r))
\end{aligned}
$$

where $\gamma(r)=h(r)-c(r)$. Once the solution of the Ornstein-Zernike equation is obtained, there are several routes to compute the thermodynamics from the pair correlation functions: the expression of the excess internal energy, the compressibility relation (Eq. 4.22) and the equation of state for thermodynamic pressure (Eqs. 4.11) or 4.16). As usual, the approximate nature of the approach leads to thermodynamic inconstancy and the thermodynamic pressure obtained by thermodynamic integration of the compressibility relation

$$
\frac{\beta P}{\rho}=1-\frac{1}{\rho} \int_{0}^{\rho} d \rho^{\prime} \rho^{\prime} \int_{\Sigma} d S c\left(r, \rho^{\prime}\right),
$$

where $c(r, \rho)$ is the direct correlation function at density $\rho$, differs form the "virial" expression

in Eqs. 4.11 or 4.16). The numerical procedures for approximate integral equations have been given in Ref . [46] for the sphere $S_{2}$ and in Ref .44] for the hyperbolic plane $H_{2}$. Results will be discussed in Sec. V.

\section{Interaction potentials}

We still restrict ourselves to pairwise additive interactions and spherically symmetric potentials. If needed, these restrictions can be lifted, but it would lead here to unnecessary complications. We have already mentioned one subtlety concerning interactions in curved space: when the manifold in which the fluid is confined is embedded in higher-dimensional Euclidean space, distance-dependent pair potentials can either depend on the geodesic distance ("curved line of force") or the Euclidean distance, e.g. the chord between two points 
on a sphere ("Euclidean line of force"). To our knowledge, work on the latter type is however very limited[13, 49]. Note that the alternative does not arise in hyperbolic geometry which is not embeddable in Euclidean space. Another subtlety in spherical geometry, and more generally, on compact manifold, comes with the definition of interacting pairs. Two particles on a sphere (or a hypersphere) are joined by two segments of a geodesic (great circle) and can therefore interact "twice" with pair potentials associated with the two geodesic distances. For hard spherical particles, this clearly corresponds to the physical situation. For longer-range potentials and for a more formal use of "spherical boundary conditions" as a way to approach the thermodynamic limit in Euclidean space (see Sec. III] and Ref.[4]) this "double distance convention" [4, 42] is to be contrasted with the minimum image convention used for the "toroidal condition" corresponding to the standard implementation of periodic boundary conditions in Euclidean space. Note again that this feature is absent in the case of the hyperbolic plane $H_{2}$ (although the use of periodic boundary conditions will also bring in compact toroidal conditions).

Before moving on to a discussion of the nature of intermolecular forces in curved spaces, it is worth considering the restriction put on the form of the pair interaction potentials by the requirement that the various configurational integrals are well defined. In spherical geometry, the geodesic distance between two particles is bounded by $\pi R$ so that the interaction potentials $u(r)$ are meaningless for $\pi R$. (Even hard core systems make no sense if the hard-core diameter $\sigma$ in the spherical manifold is of the order of the (hyper)sphere radius $R$.) In hyperbolic geometry, a constraint arises from the exponential nature of the metric at long distances (see Sec. III and Appendix A). At large separations, the correlations die out and $g(r) \rightarrow 1$. A typical configurational integral that appears in the calculation of the excess internal energy or of the pressure in $H_{2}$ is then

$$
I=\int_{r_{c}}^{\infty} d r \sinh (\kappa r) u(r) \simeq \frac{1}{2} \int_{r_{c}}^{\infty} d r e^{\kappa r} u(r),
$$

where $r_{c}$ is chosen much larger than the radius of curvature $\kappa^{-1}$. The integral in Eq. 4.38) converges if the pair interaction decreases faster than $\exp (-\kappa r)$ at long distance. One therefore concludes that the thermodynamic limit only exists in $H_{2}$ (and higher-dimensional hyperbolic manifolds) if and only if the spherically symmetric pair potentials vanish exponentially fast with distance above the radius of curvature, i.e. faster than $\exp (-\kappa r)$. For instance, this precludes using nontruncated power-law potentials such as the celebrated 
Lennard-Jones atomic model.

After this instructive detour, let us come back to the basics of intermolecular forces [50]. On top of the short-range repulsive interactions whose origin lies in the overlap of the outer electron shells of the atoms, longer-range interactions can be derived from electrostatics, leading to Coulombic, dipolar, etc... interactions, as well as from the multipole dispersion interactions between the instantaneous electric moments in one atom and those induced in the other [50]. Roughly speaking, all the longer-range interactions can be obtained from the knowledge of the Coulomb potential. In infinite Euclidean and hyperbolic space (we consider the two-dimensional case for simplicity), the Coulomb potential $v(r)$ at a geodesic distance $r$ of a unit point charge is obtained from the Poisson equation

$$
\Delta v(r)=-2 \pi \delta^{(2)}(r, \phi),
$$

where $\Delta$ is the Laplace-Beltrami operator in polar coordinates and $\delta^{(2)}(r, \phi)$ is the Dirac distribution in the appropriate manifold. The boundary condition is that the potential vanishes at infinity. In $H_{2}$ (see Appendix A), the solution of Eq. (4.39) satisfying the boundary conditions is [51]

$$
v(r)=-\ln \left(\tanh \left(\frac{\kappa r}{2}\right)\right),
$$

which behaves as $2 \exp (-\kappa r)$ as $\kappa r \rightarrow \infty$.

In spherical geometry, the Poisson equation, has no acceptable solution as it leads to a singularity for particles on opposite poles. A way out is to define the Coulomb potential for a "pseudo-charge" corresponding to a unit positive point charge and a uniform background of total charge -1 [5, 52, 55] (another definition consists in taking a +1 point charge and a -1 point charge located at the antipodal position[55]). The resulting potential on a sphere $S_{2}$ of radius $R[5]$ is

$$
v(r)=-\ln \left(\sin \left(\frac{r}{2 R}\right)\right) .
$$

Note that the hyperbolic and spherical expressions of the Coulomb potential converge to the two-dimensional Euclidean logarithmic potential, $-\ln (r / c s t)$, when the curvature goes to zero. (The extension of Eq. (4.41) to the hypersphere $S_{3}$ is given in Ref.[5, [56]).)

The above expressions of the Coulomb potential in curved space indicate the direction that one should take to properly define interatomic forces in the "curved line-of-force" interpretation. In the hyperbolic geometry, for which this is crucial, one should envisage multipolar 
and dispersion interactions as generated by appropriate derivatives of the Coulomb potential. As a consequence, all such pair interactions decrease exponentially at long distance as $\exp (-\kappa r)$ or faster. Consider for instance a dipole made of two opposite charges $\pm q$ located at positions $\mathbf{r}_{1}$ and $\mathbf{r}_{2}$. The distance separating the two charges is denoted by $d$. By using the relation between geodesic distances (see Appendix A) and taking the limit when $d \rightarrow 0$, $q \rightarrow \infty$ with $p=q d$ finite, one obtains

$$
v_{d}(r, \phi)=-\frac{p \cos (\phi)}{\sinh (\kappa r)}
$$

where $p$ is the dipole moment an $\mathrm{d} \phi$ the angle between the dipole moment and the direction of the geodesic joining the position of the dipole and the center $O$. A crude reasoning, based on a one-dimensional Drude model, suggests that dispersion forces give rise to a pair interaction potential that goes as

$$
u(r) \sim-\frac{\cosh (\kappa r)^{2}}{\sinh (\kappa r)^{4}}
$$

Therefore, at short distance, the potential behaves as $1 / r^{4}$, as expected in two-dimensional Euclidean space, whereas decays exponentially as $\exp (-2 \kappa r)$ when the distance $r$ is larger than the radius of curvature $\kappa^{-1}$. In Euclidean space, it is known that the simple Drude model captures the leading dependence on $r$, more sophiscated and realistic treatments only changing the prefactor of the interaction.

If one is interested in studying generic properties of simple liquid models in hyperbolic space, with no direct connection to realistic interatomic forces, one should make sure, at least, that the model pair potentials are truncated beyond some cut-off so that the longdistance behavior remains well defined.

\section{E. Computer simulations}

Computer simulations play a major role in liquid-state studies in Euclidean space. Unsurprisingly, they have also been developed for investigating fluid and condensed phases in curved space. Monte Carlo simulations for short-range 13, 40,42, 57 62, and longrange Coulombic-like[52, 53, 63, 64] potentials in two and three-dimensional geometry, and more seldom Molecular Dynamic studies in two-dimensional spherical 65 67] and hyperbolic manifolds 31, 32, 68 70, have been performed . Early on, numerical packing protocols have 
also been considered in spherical[71, 72] and hyperbolic[11] geometries. In this section, we do not intend to give details on the methods that have been implemented but rather to stress the differences with the Euclidean case and the technical difficulties encountered.

A simplification coming with spherical backgrounds is that there is no need for periodic boundary conditions as the whole space is closed and finite. As already mentioned, this is the rationale underlying the use of "spherical boundary conditions" in order to approach the thermodynamic limit in Euclidean space. The price to pay is that in Monte Carlo algorithms one must ensure a proper sampling of the (positively) curved manifold, with e.g. no bias toward the poles or the equator [40], and in Molecular Dynamic studies, one has to be careful with the equations of motion in curved space. The technical difficulties however are reasonable.

The case of the hyperbolic plane $H_{2}$ (higher-dimensional hyperbolic manifolds have not been studied by computer simulation), which, contrary to spherical manifolds, cannot be embedded in higher-dimensional Euclidean space, is much trickier. First, parallel transport in $H_{2}$ is complex, and implementing an algorithm for solving the equations of motion either for hard-core particles (where collisions must be handled[31, 32]) or continuous interaction potentials (where forces must computed and vector quantities added and transported [68, 69]) is by no means obvious. However, the main challenge lies in the necessary use of boundary conditions. As discussed in Sec. III and further developed in the preceding subsections IV A, IV B, IV C, one is indeed interested in the "bulk" behavior of liquids in the hyperbolic plane. In a simulation that is anyhow constrained to finite systems, the only way to limit the boundary effects, which are expected to be strong in hyperbolic geometry (see Sec. III), is to implement periodic boundary conditions 34. This amounts to choosing a primitive cell (which contains the physical system) such that it can be infinitely replicated to tile the whole plane. To ensure smoothness and consistency, the edges of the cell must be paired in a specific way. In $H_{2}$, the smallest such primitive cell equipped with an edge-pairing (or "fundamental polygon") is an octagon. As the area of the primitive cell is fixed for a given choice of boundary condition, one must change the latter, and increase the number of edges of the fundamental polygon, to study finite-size effects at constant curvature and particle density[34]. This of course does not occur in Euclidean space where, due to the absence of any metric-related length scale, the system size can be changed while keeping the same type of boundary condition. Hyperbolic boundary conditions have been developed and discussed 
in Ref. 34] and implemented in Molecular Dynamics in Refs. 68 70]. More details are given in Appendix B.

\section{F. Self-motion and diffusion equation}

Up to this point, we have focused on static properties. We now briefly consider aspects pertaining to the dynamics of fluids in curved manifolds, more specifically the self-diffusion

of particles. In Euclidean space, the self-motion of the particles in a liquid is described by space-time correlation functions. The simplest one is the so-called self-intermediate scattering function $F_{s}(k, t)$ which is the Fourier transform of the self part of the densitydensity time correlation function (or self van Hove function $G_{s}(r, t)$ ) [39]),

$$
F_{s}(k, t)=\frac{1}{N} \sum_{j=1}^{N}\left\langle e^{i \mathbf{k}\left(\mathbf{r}_{j}(t)-\mathbf{r}_{j}(0)\right)}\right\rangle=\frac{1}{N} \sum_{j=1}^{N}\left\langle\cos \left(k d_{j}(0, t)\right)\right\rangle,
$$

where $d_{j}(0, t)$ is the distance traveled by atom $j$ during times 0 and $t$ and the bracket denotes an equilibrium canonical average[39]. When $k \rightarrow 0$ and $t \rightarrow \infty, F_{s}(k, t)$ is well described by a Gaussian, corresponding to a truncated cumulant expansion, exp $\left[\frac{-k^{2}}{2 d}\left\langle d(0, t)^{2}\right\rangle\right]$, and in the same long wavelength and long time limit, one expects that the self van Hove function obeys a diffusion equation similar to Fick's macroscopic law, which leads to $F_{s}(k, t)=\exp \left[-k^{2} D t\right]$ with $D$ the coefficient of diffusion [39]. Identification of the two expressions gives the Einstein relation,

$$
2 d D=\lim _{t \rightarrow \infty}\left\{\frac{\left\langle d(0, t)^{2}\right\rangle}{t}\right\} .
$$

The generalization of the self-intermediate scattering function in spherical and hyperbolic geometries makes use of the appropriate extensions of the Fourier transform (see Sec. IV C). For $S_{2}$, one finds

$$
F_{s}(k, t)=\frac{1}{N} \sum_{j=1}^{N}\left\langle P_{k R}\left(\cos \left(\frac{d_{j}(0, t)}{R}\right)\right)\right\rangle,
$$

where $k R \in \mathbb{N}$, and on $H_{2}$

$$
F_{s}(k, t)=\frac{1}{N} \sum_{j=1}^{N}\left\langle P_{-\frac{1}{2}+i \frac{k}{\kappa}}\left(\cosh \left(\kappa d_{j}(0, t)\right)\right)\right\rangle .
$$

We recall that $P_{k R}(x)$ is a Legendre polynomial and $P_{-\frac{1}{2}+i \frac{k}{\kappa}}(x)$ is a Legendre function of the first kind, while $d_{j}(0, t)$ is the geodesic distance traveled by atom $j$ between 0 and $t$ and calculated with the appropriate metric. 
One may then wonder what is the small $k$ and long time limit of the intermediate scattering function in non-Euclidean space. One expects that it converges to a result predicted by the associated diffusion equation,

$$
\frac{\partial G_{s}(r, t)}{\partial t}=D \Delta G_{s}(r, t)
$$

with $\Delta$ the Laplace-Beltrami operator acting in the curved manifold. (Actually, by isotropy one expects $G_{s}$ to only depend on $r$ so that only the "radial" part of the operator is required.) We keep focusing on the two-dimensional manifolds $S_{2}$ and $H_{2}$ for simplicity. The diffusion problem has been solved in both cases with the solution in $S_{2}$ given by 73

$$
G_{s}^{\left(S_{2}\right)}(r, t)=\sum_{k=0}^{\infty}\left(\frac{2 k+1}{k}\right) \sin \left(\frac{r}{R}\right) P_{k}\left(\cos \left(\frac{r}{R}\right)\right) e^{-\frac{k(k+1) D t}{R^{2}}}
$$

and that in $H_{2}$ by [74]

$$
G_{s}^{\left(H_{2}\right)}(r, t)=\frac{e^{-\frac{\kappa^{2} D t}{4}}}{2 \sqrt{2 \pi}(D t)^{\frac{3}{2}}} \sinh (\kappa r) \int_{r}^{\infty} d y \frac{y e^{-\frac{y^{2}}{4 D t}}}{\sqrt{\cosh (\kappa y)-\cosh (\kappa r)}} .
$$

One can check that when the curvature goes to zero, i.e. $R \rightarrow+\infty$ in Eq. 4.49) and $\kappa \rightarrow 0$ in Eq. 4.50, both expressions reduce to the standard two-dimensional Euclidean formula,

$$
G_{s}^{\left(E_{2}\right)}(r, t)=\frac{e^{-\frac{-r^{2}}{4 D t}}}{4 \pi D t}
$$

Generalizations to the three-dimensional manifolds $S_{3}$ and $H_{3}$ are given in Ref. [73, 75] and Ref.[74], respectively.

Diffusion on a sphere (or a hypersphere) is such that the particles can never escape from the closed space they are embedded in and, as a result, the mean square displacement $\left\langle d(0, t)^{2}\right\rangle$ goes to a constant value when $t \rightarrow+\infty$. Procedures to fit simulation data for extracting a self diffusion coefficient from the $\left\langle d(0, t)^{2}\right\rangle$ have been given in Refs. [73, 75]. Diffusion on Riemann manifolds (with a non constant curvature) has been recently investigated in the short-time limit[76]. For the hyperbolic plane, the diffusion motion at long times is characterized by two regimes: when the mean traveled distance is large, but smaller than the radius of curvature $\kappa^{-1}$, one has an ordinary diffusion regime with $\left\langle d(0, t)^{2}\right\rangle \sim D t$ and $\langle d(0, t)\rangle \sim \sqrt{D t}$; on the other hand, when the mean traveled distance is large compared to $\kappa^{-1}$, one finds a "ballistic" diffusion regime with $\left\langle d(0, t)^{2}\right\rangle \sim(D t)^{2}$ and $\langle d(0, t)\rangle \sim D t$ [74, 77]. 


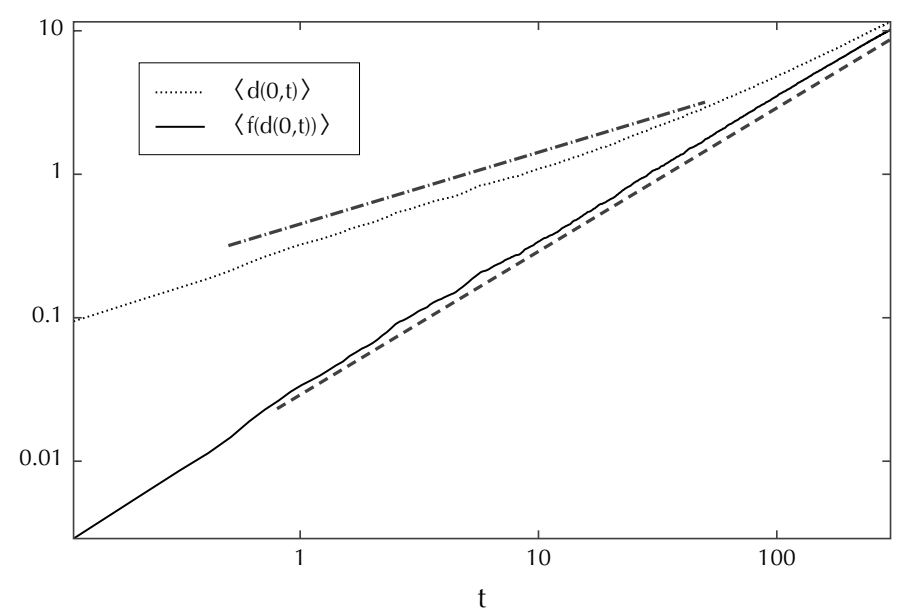

FIG. 1: Log-log plot of the mean absolute displacement $\langle d(0, t)\rangle$ in units of $\kappa^{-1}$ and of $\langle f(d(0, t))\rangle$ (see Eq. (4.52)). The dashed line has a slope equal to 1 and the dotted-dashed, a slope of $1 / 2$. To good approximation, $\langle f(d(0, t))\rangle$ has a linear time dependence at all times, which corresponds to a diffusive regime in the hyperbolic sense. The parameters are $\rho \sigma^{2}=0.91, T=2.17$, and $\kappa \sigma=0.2$. The system with octagonal periodic boundary conditions comprises 287 atoms.

It has been shown in Ref.[77] that the self-diffusion coefficient can be conveniently extracted from simulation data of the function $\frac{1}{N} \sum_{j=1}^{N}\left\langle f\left(d_{j}(0, t)\right)\right\rangle$ where

$$
f(r)=\kappa^{-2} \ln \left(\frac{1+\cosh (\kappa r)}{2}\right) .
$$

Indeed, when calculated with the solution of the hyperbolic diffusion equation, this function behaves as $D t$ for both the ordinary and ballistic diffusion regimes. In Fig. 1, we show the Molecular Dynamics simulation result for a fluid with truncated Lennard-Jones interactions on $\mathrm{H}_{2}$ (see below) [77] at a rather high temperature. It is clearly found that the atomic motion becomes diffusive at long enough time, and the self-diffusion coefficient can be determined without having to worry about the crossover between ordinary and ballistic diffusion. 


\section{CURVATURE EFFECT ON THE THERMODYNAMICS AND STRUCTURE OF SIMPLE FLUIDS}

\section{A. Short-range interaction potentials}

In order to illustrate the influence of a nonzero curvature on the properties of a fluid embedded in a non-Euclidean space, we begin with the two dimensional hard-disk fluid on a sphere $S_{2}$ of radius $R$ and a hyperbolic plane $H_{2}$ of radius of curvature $\kappa^{-1}$. (We recall that the Gaussian curvature $K$ is constant in both cases and equal to $1 / R^{2}$ for $S_{2}$ and $-\kappa^{-2}$ for $H_{2}$.) The "virial" equation of state giving the thermodynamic pressure, i.e. the spreading pressure for $S_{2}$ and the bulk pressure for $H_{2}$ (see preceding sections), is obtained from Eqs. (4.11) and (4.16) after using the fact that for hard disks of diameter $\sigma$ in curved space, $\frac{d}{d r} \exp (-\beta u(r))=\delta(r-\sigma)$. For $S_{2}[4,13$,

$$
\left.\frac{\beta P}{\rho}\right|_{S_{2}}=1+\rho \frac{\pi R^{2}}{2}\left(\frac{\sigma}{R}\right) \sin \left(\frac{\sigma}{R}\right) g\left(\sigma^{+}\right),
$$

and for $H_{2}$ [44]

$$
\left.\frac{\beta P}{\rho}\right|_{H_{2}}=1+\rho \frac{\pi}{2 \kappa^{2}}(\cosh (\kappa \sigma)-1) g\left(\sigma^{+}\right),
$$

where in both cases $g\left(\sigma^{+}\right)$is the radial distribution function at contact.

In the low density limit, $g\left(\sigma^{+}\right)$goes to 1 and the second viral coefficient (see also Eqs. 4.18) and (4.19) ) reads

$$
\left.B_{2}\right|_{S_{2}}=\frac{\pi R^{2}}{2}\left(\frac{\sigma}{R}\right) \sin \left(\frac{\sigma}{R}\right),
$$

and

$$
\left.B_{2}\right|_{H_{2}}=\frac{\pi}{\kappa^{2}}(\cosh (\kappa \sigma)-1),
$$

where we have considered $N \gg 1$ for $S_{2}$ and Eq. (5.3) is only defined for $\sigma<\pi R$. For $H_{2}$, even if nothing a priori prevents one from taking the particle diameter much larger than $\kappa^{-1}$, the discussion in Sec. IVD indicates that the physical interactions decrease exponentially with distance beyond $\kappa^{-1}$. Particle diameters $\sigma \gg \kappa^{-1}$, which lead to an exponentially growing $\left.B_{2}\right|_{S_{2}}$, have therefore no physical significance. Higher-order virial coefficients have been calculated for $S_{2}$ [4, 13] and for $H_{2}\left[31\right.$. (The three-dimensional spherical case $S_{3}$ has been considered in Refs. [40, 41, 59].)

As already noted, there is no simple symmetry $\kappa \leftrightarrow i R^{-1}$ between the $H_{2}$ and $S_{2}$ pressure expressions, due to the finite size of the sphere whose total area can only be changed by 
varying the curvature. For instance, when the curvature goes to zero in $S_{2}$ and $H_{2}$, the equation of state and virial coefficients approach the Euclidean result, albeit in a nonsymmetric way. As an illustration,

$$
\begin{gathered}
\left.B_{2}\right|_{S_{2}}=\left.B_{2}\right|_{E_{2}}\left(1-\frac{1}{6} K \sigma^{2}+O\left(K^{2} \sigma^{4}\right)\right), \\
\left.B_{2}\right|_{H_{2}}=\left.B_{2}\right|_{E_{2}}\left(1+\frac{1}{12} K \sigma^{2}+O\left(K^{2} \sigma^{4}\right)\right),
\end{gathered}
$$

where $\left.B_{2}\right|_{E_{2}}=\pi \sigma^{2} / 2$. The asymmetry between $S_{2}$ and $H_{2}$ is not compensated if one express the virial expression in terms of the packing fraction $\eta$ (or rather "surface coverage" in two dimensions) instead of the density $\rho$. Indeed,

$$
\begin{aligned}
& \left.\eta\right|_{S_{2}}=2 \pi R^{2}\left(1-\cos \left(\frac{\sigma}{2 R}\right)\right) \rho, \\
& \left.\eta\right|_{H_{2}}=\frac{2 \pi}{\kappa^{2}}\left(\cosh \left(\frac{\kappa \sigma}{2}\right)-1\right) \rho,
\end{aligned}
$$

so that to first order,

$$
\left.\frac{\beta P}{\rho}\right|_{S_{2}}=1+\eta\left(\frac{\sigma}{2 R}\right) \frac{\sin \left(\frac{\sigma}{2 R}\right) \cos \left(\frac{\sigma}{2 R}\right)}{1-\cos \left(\frac{\sigma}{2 R}\right)}+O\left(\eta^{2}\right)
$$

and

$$
\left.\frac{\beta P}{\rho}\right|_{H_{2}}=1+\eta \frac{\sinh \left(\frac{\kappa \sigma}{2}\right)^{2}}{\cosh \left(\frac{\kappa \sigma}{2}\right)-1}+O\left(\eta^{2}\right) .
$$

To go beyond the low-density expansion, several routes have been taken for exploring the hard-disk fluid in spherical and hyperbolic geometries. One can build approximate equations of state from scaled particle theory [49, 78], free area theory 31, 32], or by constructing rational approximants from the first virial coefficients[31, with possible additional input from some close packing density[13, 59, 79]. One can also compute the radial distribution function $g(r)$, either from approximate self-consistent integral equations [44, 46] of from simulations [13, 31, 32, 59, 60. The thermodynamic pressure can be obtained through the "virial", Eqs. (5.1) or (5.2), or the compressibility, Eq. (4.37), relations.

As in Euclidean space, all approximate methods, including integral equations, are limited to moderate densities, i.e. densities that are less than the ordering transition in the Euclidean plane. The exact location, the nature and the order of the latter are known to be strongly system-size dependent (see Sec. VI), so we typically mean here densities $\rho \sigma^{2} \lesssim 0.8$ and packing fractions $\eta \lesssim 0.6$. In Fig. 2 , we compare for the case of the hyperbolic plane 


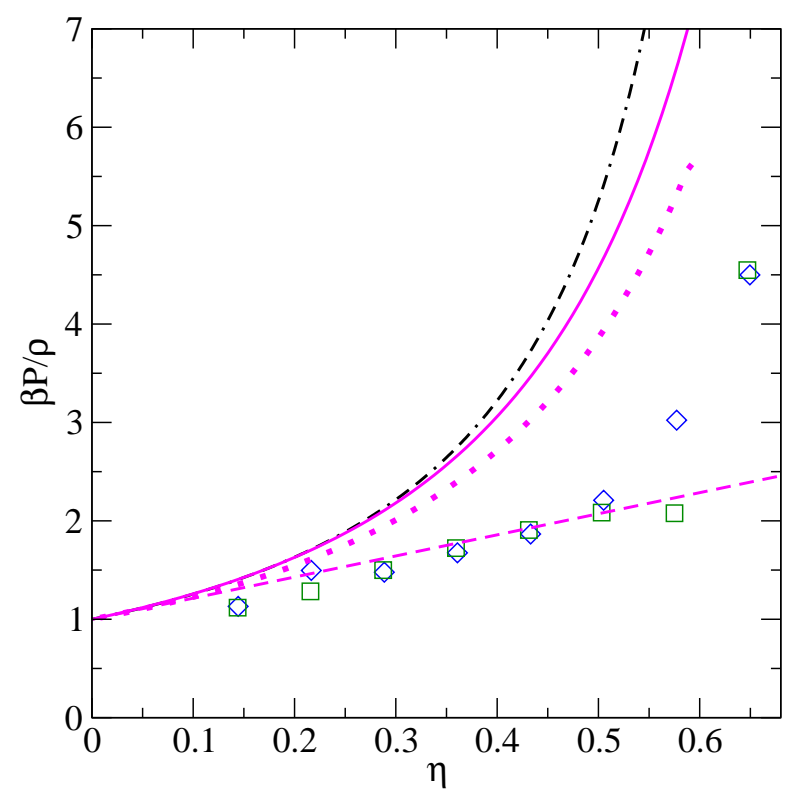

FIG. 2: Equation of state of the hard-disk fluid in $H_{2}$ versus packing fraction $\eta$ for a quite strong curvature $\kappa \sigma \simeq 1.06$. Comparison between the PY results (compressibility route (dotted line) and virial route (full line)) for $\kappa \sigma=1.06$ and the simulation results of Ref. [31] for $\kappa \sigma=1.060$ (green squares) and $\kappa \sigma=1.062$ (blue diamonds). The dashed-dotted line is the prediction of Ref. [79] and the dashed line corresponds to the equation of state truncated at the second virial coefficient. The disagreement between predictions and simulation data is significant alrealdy for $\eta \simeq 0.2$.

with a quite strong curvature $\kappa \sigma \simeq 1.06$ the equations of state obtained by simulation [31, 32] and predicted by several approximate methods (approximate equations of state [31, 32, 79] and results from the Percus-Yevick integral equation with both the virial and the compressibility routes [4] As in the Euclidean case, the compressibility route gives a higher bulk thermodynamic pressure than the virial route. An empirical recipe that has proven very accurate in Euclidean space 39] is to consider a linear combination

$$
P=\frac{2 P_{c}+P_{v}}{3}
$$

where $P_{c}$ and $P_{v}$ are the "compressibility" and "virial" pressures. In Fig. 2, one can see that all predictions, except for the free-area method (which for this reason not reproduced 


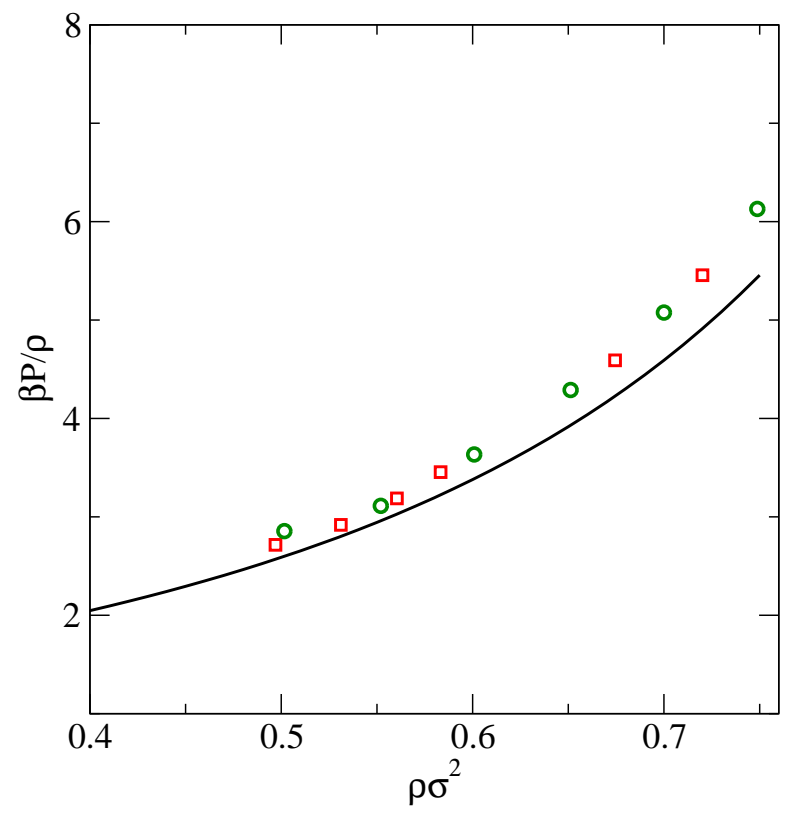

FIG. 3: Equation of state of the hard-disk fluid in $S_{2}$ and in $H_{2}$ for the same magnitude of the curvature $\kappa \sigma=\frac{\sigma}{R} \simeq 0.45$. The symbols denote simulation results in $S_{2}$ (squares) and in $E_{2}$ (circles). The full curve corresponds to the PY approximation in $H_{2}$. The PY result in $S_{2}$ [46] is virtually indistinguishable from the simulation results (on this scale).

here), approach at low density the exact expression truncated at the second virial coefficient, Eq. (5.9), whereas their validity rapidly deteriorates for even moderate coverage. One should however keep in mind that the corresponding simulations 31, 32 have been carried out with a very small number of atoms, always less than 10, and that the curvature is quite strong.

For the spherical substrate $S_{2}$, most of the existing data concern the approach to the Euclidean plane by following the equation of state $\beta P / \rho$ versus $\eta$ or $\rho \sigma^{2}$ at constant number $N$ of atoms (which implies that the curvature varies with density, as $\rho \sigma^{2}=\left(\frac{N}{4 \pi}\right)\left(\frac{\sigma}{R}\right)^{2}$ ). We nonetheless display in Fig. 3 a comparison between the equations of state obtained from the the Percus-Yevick integral equation via the compressibility route for the hard-disk fluid in $S_{2}$ [46] and $H_{2}$ [44] for the same "radius of curvature" $\sigma / R=\kappa \sigma=0.45$, as well as simulation results in $S_{2}$ and $E_{2}$. Despite possible limitations of the Percus-Yevick approximation, it appears that the results in $S_{2}$ almost coincide with those in the Euclidean $E_{2}$ whereas those in $H_{2}$ are significantly different for $\rho \sigma^{2} \gtrsim 0.55$. 


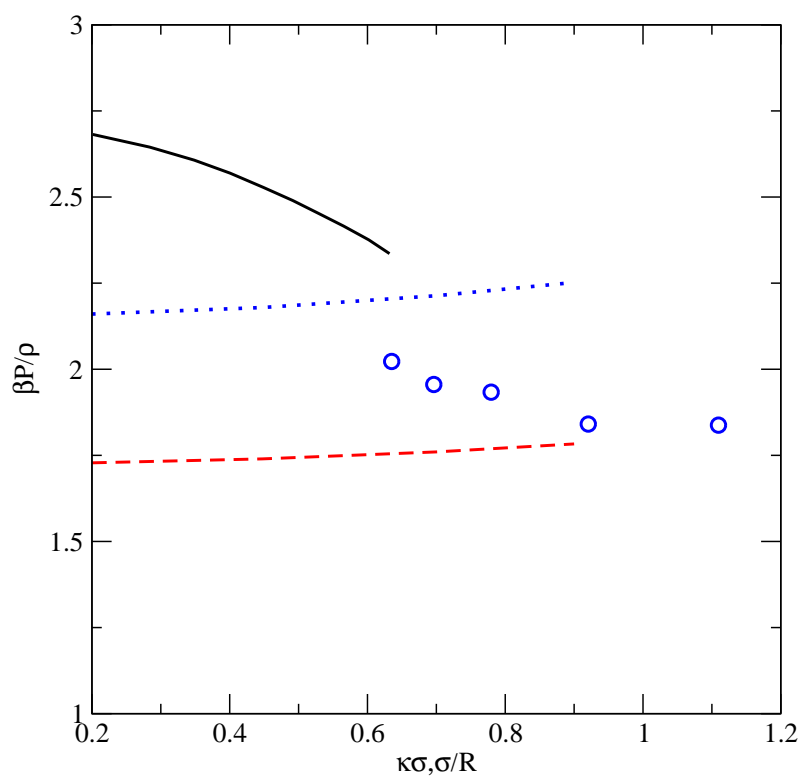

FIG. 4: Compressibility factor $\beta P / \rho$ of the hard-disk fluid in $S_{2}$ and $H_{2}$ as a function of the curvature parameter, $\sigma / R$ or $\kappa \sigma$. For $S_{2}$, are plotted the result from the PY approximation for $\rho \sigma^{2}=0.8[46$ (full line, where for convenience $(\beta P / \rho-5)$ is plotted) and the simulation data for $\eta=0.4$ (which corresponds to $\rho \sigma^{2}$ varying between 0.385 and 0.392

[13] (symbols). For $H_{2}$, the two curves are obtained from the PY approximation with $\rho \sigma^{2}=0.2$ (dotted line) and $\rho \sigma^{2}=0.4$ (dashed line).

In addition, to illustrate the influence of curvature, i.e. both its magnitude and its sign, on the thermodynamic properties of a fluid, we have collected in Fig. 4 simulation data and integral-equation results for the compressibility factor $\beta P / \rho$ of hard disks on $S_{2}$ and $H_{2}$, which we plot as a function of increasing curvature, $|K|^{-1 / 2} \sigma$, for different values of $\rho \sigma^{2}$. Whereas the pressure increases with curvature in $S_{2}$ for a given density $\rho \sigma^{2}$ (or packing fraction $\eta$ ), the opposite trend is observed in $H_{2}$. This effect can already be seen as very small densities when considering only the correction to the ideal-gas behavior due to the second virial coefficient (see above).

The main advantage of computing the radial distribution function $g(r)$ is that it also provides some information on local structural order in the fluid. We focus here on the fluid or liquid regime at densities and temperatures (for continuous interaction potentials) such 
that the system in flat space has not undergone its ordering transition (and if attractive interactions are present, away from the gas-liquid critical point). Higher densities and lower temperatures, which correspond to interesting new physics, will be considered in Sec: $\mathrm{VI}$ and VII, and critical behavior in Sec. VC. In the fluid/liquid regime at "moderate" densities and temperatures (the qualifier "moderate" having the above discussed meaning), it is found that curvature has only a weak influence, at least when the curvature is not too large, i.e. when $\sqrt{|K|} \sigma$ is significantly less than 1 . This can already be inferred from the thermodynamic data (see above). We illustrate the effect of a negative curvature on the $g(r)$ of the harddisk fluid, as obtained from the Percus-Yevick equation, in Fig. 5[44]. For a packing fraction $\eta=0.55$, it is found that the curves are essentially superimposable on the Euclidean one for a range of curvature parameter $\kappa \sigma$ between 0 and 0.5 . Only when going to higher curvatures, e.g. $\kappa \sigma=1.5$, can one distinguish the influence of the curvature in the structure. (Note that on the sphere, strong curvatures with $\sigma \gtrsim R$ correspond to small systems for which finite-size and discreteness effects are very important[13].)

The same conclusion about the curvature effect has been reached for a truncated LennardJones model in $\mathrm{H}_{2}$, with no detectable changes from flat-space results up to $\kappa \sigma \simeq 0.5$ [44]. The model is defined by a pair potential

$$
u(r)=4 \epsilon\left[\left(\frac{\sigma}{r}\right)^{12}-\left(\frac{\sigma}{r}\right)^{6}\right]+u_{c}
$$

that is truncated for $r \geq r_{c}$ with $r_{c}=2.5 \sigma ; u_{c}$ is the shift obtained from the relation $u\left(r_{c}\right)=0$. In this study, a large range of temperature, density and curvature parameter has been investigated through the solution of the Percus-Yevick and HNC integral equations. As far as we know, this is also the only work in which the validity of such integral equations in curved space has been tested. As shown in Fig. 7, of Ref. [44], the predictions compare well with Molecular Dynamics simulation data with a slight advantage to the Percus-Yevick approximation.

\section{B. Coulombic systems}

We have briefly reviewed in Sec. IVD the way to define the Coulomb potential in curved space (following a "curved line of force" interpretation) via the solution of the relevant Poisson equation. We have also stressed the difference between hyperbolic geometry where space 


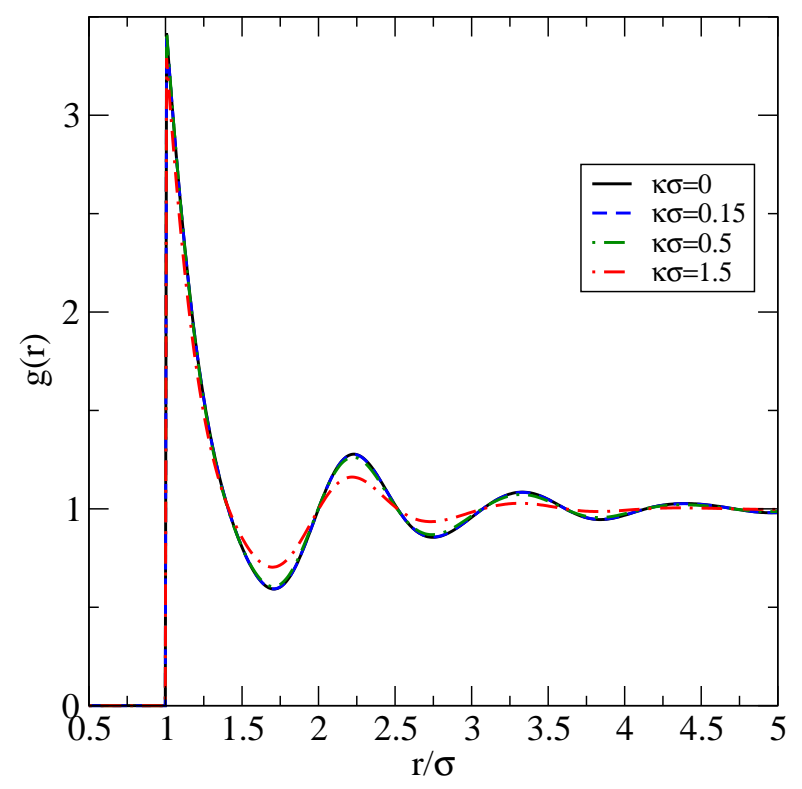

FIG. 5: Radial distribution function $g(r)$ of the hard-disk fluid in $H_{2}$, as obtained from the PY equation, at a packing fraction $\eta=0.55$ for various values of the curvature: $\kappa \sigma=0,0.15,0.5,1.5$. Only for $\kappa=1.5$, namely a radius of curvature smaller than the particle diameter, does the structure display significant deviations from the Euclidean case.

can be infinite and spherical geometry in which the finiteness of space requires the introduction of pseudo-charges in order to properly define the Coulomb potential. The main advantage of spherical geometry being the absence of boundary, "spherical" and "hyperspherical" boundary conditions have been widely used by Caillol, Levesque and coworkers [5, 6, 5255, 63, 64] to approach the thermodynamic limit of Coulombic systems in Euclidean space (as the radius of the sphere or the hypersphere goes to infinity). Multipolar interactions deriving from the Coulomb potential have also been considered [53]. Here, as in most of the article, we focus on two-dimensional manifolds of constant positive $\left(S_{2}\right)$ or negative $\left(H_{2}\right)$ curvature and we consider systems of charges interacting through the Coulomb potential,

$$
\begin{gathered}
\left.u(r)\right|_{S_{2}}=-q_{1} q_{2} \ln \left(\sin \left(\frac{r}{2 R}\right)\right), \\
\left.u(r)\right|_{S_{2}}=-q_{1} q_{2} \ln \left(\tanh \left(\frac{\kappa r}{2}\right)\right),
\end{gathered}
$$


for two charges $q_{1}, q_{2}$ (or pseudo-charges in $S_{2}$ ) separated by a geodesic distance $r$ (with $r \leq \pi R$ in $\left.S_{2}\right)$

The two simplest Coulombic systems are the one-component plasma (OCP) and the two-component plasma (TCP). The former is a monodisperse system of point charges of equal sign and magnitude $q$ embedded in a charged uniform background that maintains global electroneutrality. Conventionally, one uses the coupling constant $\Gamma=\beta q^{2}$ as control parameter. At small and intermediate coupling, $\Gamma=O(1)$ or less, the system is in a fluid phase whereas at zero temperature $(\Gamma \rightarrow+\infty)$, its ground state in the Euclidean plane is a hexagonal crystal forming a triangular lattice. The questions which remain debated[67] are (i) whether there is a freezing transition at some large but finite coupling and (ii) if indeed freezing occurs, whether the transition is a first-order one or proceeds via two continuous transitions separated by a hexatic phase as predicted by the KTNHY theory [9, 80, 81]. As will be more extensively discussed in Secs.VI and VII, placing the system in curved geometry "frustrates" hexagonal order by forcing in an irreductible number of topological defects. We just mention here that it has then been argued that approaching the thermodynamic limit of the OCP in the Euclidean plane via spherical boundary conditions avoids the artifacts generated by periodic boundary conditions on the ordering behavior [67.

The TCP consists of a binary mixture of oppositely charged point particles (with charges $\pm q$ ) in equal concentration. No neutralizating background is needed. In the Euclidean plane, the system is stable in a conducting phase up to a coupling $\Gamma=2[82,83$. Above this value, a collapse of pairs of opposite charges occurs. This can be regularized by introducing an additional hard-core repulsion (the system is then also known as the restricted primitive model). The system then undergoes a continuous Kosterlitz-Thouless transition at $\Gamma=4$ to a dielectric phase in which all charges are bounded into dipoles in the limit of an infinitesimal particle density. For larger densities, the critical temperature of the Kosterlitz-Thouless transition decreases and terminates in a first-order transition slightly above the gas-liquid critical point. The location of the latter remains quite difficult to obtain precisely, as both the temperature and the density are very small[84].

In the following, we restrict ourselves to the OCP and TCP in their fluid, conducting phase at small and intermediate coupling in spherical $\left(S_{2}\right)$ and hyperbolic geometry $\left(H_{2}\right)$. Several properties found in the Euclidean plane carry over to $S_{2}$ and $H_{2}$ : (i) the small coupling/high temperature regime is (asymptotically) described by the linearized Debye- 
Hückel approximation, (ii) exact solutions for the thermodynamics and the structure are obtained for the special value of the coupling constant $\Gamma=2$, and (iii) in the conducting phase, exact sum rules generalizing the Stillinger-Lovett relations are satisfied.

We begin with the OCP. Assuming a perfect compensation between the point charges and the background amounts to replacing $g(r)$ by the pair correlation function of the point charges, $h(r)=g(r)-1$, in the equation of state for the thermodynamic pressure, Eqs.(4.11) and (4.16). After inserting Eqs.(5.13) and (5.14), one finds

$$
\begin{aligned}
& \left.\frac{\beta P}{\rho}\right|_{S_{2}}=1+\rho\left(\frac{\pi \Gamma}{4}\right) \int_{0}^{\pi R} d r r\left(1+\cos \left(\frac{r}{R}\right)\right) h(r), \\
& \left.\frac{\beta P}{\rho}\right|_{H_{2}}=1+\rho\left(\frac{\pi \Gamma}{\kappa}\right) \int_{0}^{\infty} d r\left(\frac{\cosh (\kappa r)-1}{\sinh (\kappa r)}\right) h(r),
\end{aligned}
$$

which both converge to the Euclidean formula

$$
\left.\frac{\beta P}{\rho}\right|_{E_{2}}=1+\rho\left(\frac{\pi \Gamma}{2}\right) \int_{0}^{\infty} d r r h(r)
$$

when the curvature goes to zero. It should be stressed that defining a pressure for the OCP is far from trivial as both the way one treats the uniform background and the choice of embedding conditions may matter. This has been carefully discussed in the case of the hyperbolic plane $H_{2}$ for which no less than 5 different pressures have been considered[45]. The above defined "bulk thermodynamic pressure" reduces to the standard thermodynamic pressure (then also equal to the "Maxwell pressure") with vanishing curvature.

It has been shown for $S_{2}[85]$ and $H_{2}[51$ that the OCP in the conducting phase satisfies generalized Stillinger-Lovett sum rules[86],

$$
\rho \int_{S_{2} \text { or } H_{2}} d S h(r)=-1,
$$

which expresses the strictly enforced electroneutrality, and

$$
\begin{aligned}
& \pi R^{2} \rho^{2} \Gamma \int_{S_{2}} d S h(r)\left(1-\cos \left(\frac{r}{R}\right)\right)=-1 \\
& \frac{4 \pi \rho^{2} \Gamma}{\kappa^{2}} \int_{H_{2}} d S h(r) \ln \left(\cosh \left(\frac{\kappa r}{2}\right)\right)=-1,
\end{aligned}
$$

which both express the screening property, with $\sqrt{2 \pi \rho \Gamma}$ the inverse of the Debye screening length. Note that the sum rules in the case of $H_{2}$ should be interpreted as valid in the bulk (see Secs. III and IV and Ref. [87]). 
In the high-temperature or small-coupling limit, the linearized Debye-Hückel approximation becomes asymptotically exact and analytical expressions for the pair correlation function can be derived. The direct correlation function $c(r)$ becomes equal to $-\beta u(r)$, so that by using the Ornstein-Zernike relation, Eq. 4.32, one obtains in $S_{2}$

$$
h(\theta)=-\Gamma\left(\frac{1}{2\left(\chi^{2}+1\right)}+\sum_{k=1}^{\infty}\left(\frac{2 k+1}{2}\right) \frac{1}{\chi^{2}+k(k+1)} P_{k}(\cos (\theta))\right) .
$$

(No explicit expression of $h(\theta)$ has been obtained, contrary to the case of the hypersphere [5].)

In $\mathrm{H}_{2}$, one finds [51, 87]

$$
h(r)=-\Gamma Q_{\nu}(\cosh (\kappa r))
$$

where

$$
\nu=-\frac{1}{2}+\sqrt{\frac{1}{4}+\frac{2 \pi \rho \Gamma}{\kappa^{2}}} .
$$

As anticipated, one can show that the above Debye-Hückel expressions satisfy the generalized Stillinger-Lovett sum rules[51]. Eqs.5.21 and 5.22 can then be inserted in Eqs. (5.15) and (5.16) to derive the equation of state[44]. For illustration, we consider the $\Gamma \rightarrow 0$ limit in the hyperbolic plane. At fixed curvature, Eq. (5.23) shows that $\nu \rightarrow 0$ and, by using the definition $Q_{0}(x)=\frac{1}{2} \ln \left(\frac{1+x}{1-x}\right)$ and the property that $\int_{1}^{\infty} d x \frac{Q_{0}(x)}{1+x}=\frac{\pi^{2}}{3}$, one finally obtains that 44

$$
\left.\frac{\beta P}{\rho}\right|_{H_{2}} \simeq 1-\left(\frac{\rho \pi^{3} \Gamma^{2}}{12 \kappa^{2}}\right)
$$

as $\Gamma \rightarrow 0$. On the other hand, at fixed (small) $\Gamma$ and $\kappa \rightarrow 0$, one finds that the Debye-Hückel expression in $\mathrm{H}_{2}$ leads to

$$
\left.\frac{\beta P}{\rho}\right|_{H_{2}}=1-\frac{\Gamma}{4}
$$

which is the exact result for the thermodynamic pressure. It is also worth noting that in $H_{2}$, the exponential decay of the Coulomb potential restores an analytical virial expansion in the Debye-Hückel limit for a finite radius of curvature $\kappa^{-1}$ whereas in Euclidean spaces, the pressure is independent of density in $E_{2}$ and has a nonanalytical behavior in $E_{3}[88]$.

As mentioned above, for the specific value of the coupling constant $\Gamma=2$, exact analytical expressions of the thermodynamic quantities and the pair correlation function $h(r)$ can be obtained in Euclidean [89], spherical [85, 90], and hyperbolic geometries [51, 91] (as well as on a "Flamm's paraboloid "92]). For spherical and hyperbolic geometries, the pair correlation 
function is given by

$$
\left.h(r)\right|_{S_{2}}=-\left(\frac{1+\cos \left(\frac{r}{R}\right)}{2}\right)^{4 \pi \rho R^{2}}
$$

and

$$
\left.h(r)\right|_{H_{2}}=-\left(\cosh \left(\frac{\kappa r}{2}\right)\right)^{-2+\frac{8 \pi \rho}{\kappa^{2}}} .
$$

From the above expressions, one can calculate the thermodynamic pressure with the following results:

$$
\left.\frac{\beta P}{\rho}\right|_{S_{2}}=\frac{1}{2}
$$

and

$$
\left.\frac{\beta P}{\rho}\right|_{H_{2}}=\frac{2 \pi \rho+\kappa^{2}}{4 \pi \rho+\kappa^{2}} .
$$

Note that the low-density limit of Eq. (5.29) coincides, as it should, with Eq. (5.24) when $\Gamma=2$. When the curvature goes to zero, Eq. 5.29 reduces to the Euclidean result, $\frac{\beta P}{\rho}=$ $\frac{1}{2}$, whereas the spherical expression always coincides with it, regardless of the (positive) curvature. An interesting observation concerning the hyperbolic plane is that if one takes the limit of an infinite (negative) curvature, $\kappa \rightarrow \infty$, the pressure goes to the ideal-gas limit. This result is expected to be quite general (see Eq. (5.28) and Sec. VB): the influence of the interactions appears to vanish in the limit of large negative curvature, if however this limit makes any sense.

Having discussed the OCP in two-dimensional curved manifolds (more details can be found in Refs. [54, 85, 90] for $S_{2}$ and Refs. [44, 45, [51, 87, 91] for $H_{2}$ ), we now move on to the case of the TCP model. In this system, there is no background and one has to consider the correlation functions between pairs of equal charges, $h_{++}(r)=h_{--}(r)$, and of opposite charges, $h_{+-}(r)=h_{-+}(r)$. Electroneutrality implies

$$
\rho \int d S\left(h_{++}(r)-h_{+-}(r)\right)=-2
$$

where $\rho=2 \rho_{+}=2 \rho_{-}$is the total particle density. Higher-order Stillinger-Lovett types of sum rules are also satisfied by $h_{++}(r)$ and $h_{+-}(r)$ and the thermodynamic pressure from Eqs. 4.11) and (4.16) is now given by equations similar to Eqs. (5.15) and (5.16) with $\rho$ replaced by $\rho / 2$ and $h(r)$ by $\left(h_{++}(r)-h_{+-}(r)\right)$.

In the small coupling limit, the Debye-Hückel approximation gives the first nontrivial term in $\Gamma$. As the linearized Poisson equation is similar to that of the OCP, the pair 
correlation functions are given by $h_{++}(r)=-h_{+-}(r)$ with $h_{++}(r)+h_{+-}(r)$ equal to the solution in Eqs.(5.26) and (5.27). Consequently, the equation of state as the same of the OCP given above. Exact analytical results are also obtained for the special coupling value of $\Gamma=2$. However, the calculations become quite involved and tedious. Details concerning the hyperbolic case can be found Refs. [51, 87, 91].

\section{Liquid-gas critical behavior}

Critical behavior implies fluctuations on all spatial scales and a diverging correlation length. Strictly speaking, critical phenomena cannot exist in finite systems and therefore not for systems confined to spherical substrates. (As stressed before, spherical geometry can however be used as a trick to approach the thermodynamic limit in Euclidean space and to study finite-size scaling by decreasing the curvature and concomitantly increasing the system size[62].) On the other hand, bona fide critical points can be present in systems embedded in hyperbolic geometry as space can then be infinite. We consider in this section the gas-liquid critical behavior of atomic fluids in the hyperbolic plane $H_{2}$.

Drastic changes in critical behavior are expected in hyperbolic geometry. This is known from field theoretic studies: a negative curvature acts as an "infrared regulator", suppressing fluctuations on wavelengths larger than the radius of curvature 93. As a consequence, critical behavior in statistical systems on a hyperbolic manifold is expected to be mean-fieldlike, with classical values of the critical exponents $(\eta=0, \gamma=1$, etc) 94 96]. This mean-field character can be understood by going back to the analysis of section IVC concerning the correlation functions and the compressibility. The latter is given by an integral over space, Eq. (4.22), which in the case of $H_{2}$ is expressed as

$$
\frac{\rho \chi_{T}}{\beta}=1+\rho \frac{2 \pi}{\kappa} \int_{0}^{\infty} d r \sinh (\kappa r) h(r) .
$$

For long distances, $\sinh (\kappa r) \sim(1 / 2) \exp (\kappa r)$, and, as already pointed out in the section concerning pair interactions, the integral over the whole space $H_{2}$ is bounded if and only if $h(r)$ decreases at large $r$ faster than $\exp (-\kappa r)$. Assume for instance that $h(r)$ decreases exponentially as $\exp (-r / \xi)$ with $\xi$ the correlation length. One then finds that Eq. (5.31) becomes

$$
\frac{\rho \chi_{T}}{\beta}=\frac{(\kappa \xi)^{2}}{1-(\kappa \xi)^{2}}+\text { finite }
$$


which diverges for $\kappa \xi \rightarrow 1$ (and is infinite for $\kappa \xi>1$ ). The compressibility can therefore diverge with a finite correlation length $\xi=\kappa^{-1}$ (this is the meaning of the "infrared regulator" discussed above). The critical point being then characterized by a finite correlation length, one expects $\xi$ to be a regular function of the control parameters $T$ and $\rho$ even in the vicinity of $\left(T_{c}, \rho_{c}\right)$,

$$
\xi(T, \rho)=\kappa^{-1}-A\left(T-T_{c}\right)-B\left(\rho-\rho_{c}\right)^{2}+\ldots
$$

with $A, B>0$. As a result, along the critical isochore,

$$
\chi_{T} \sim\left|T-T_{c}\right|^{-1}, \quad T \rightarrow T_{c}^{+}
$$

and along the critical isotherm

$$
\chi_{T} \sim\left(\rho-\rho_{c}\right)^{-2}, \quad \rho \rightarrow \rho_{c}
$$

which gives the classical values of critical exponents, $\gamma=1, \delta=3$, and $\eta=0$. Note also that, at the critical point, the Fourier-Helgason transform of the pair correlation function $\tilde{h}(k)$ (see Eq. 4.30), is finite for $k=0$, which is a dramatic illustration of Eq. (4.34), and has a regular expansion in $(k / \kappa)^{2}$ when $(k / \kappa) \rightarrow 0$.

We stress that the above reasoning concerns the "bulk" behavior in $H_{2}$, that which is obtained by taking the thermodynamic limit either with a succession of periodic boundary conditions or by removing the boundary regions (see Sec. III and IV). The exponentially growing character of the hyperbolic metric otherwise induces possible "boundary transitions" 95 which we do not consider here.

The gas-liquid critical point of the truncated Lennard-Jones fluid in $H_{2}$ has been studied through the integral equation approach 44, that has been described in Sec. IV C. The results are illustrated in Figs 6 and 7 for the Percus-Yevick closure and a curvature parameter $\kappa \sigma=$ 0.5. Fig. 6 displays the inverse compressibility along different isotherms as one approaches the critical one. It is well known that approximate integral equations such as the PercusYevick one fail to capture nonclassical critical behavior when it is present, as in the Euclidean plane. The relevant conclusions that can be drawn from the Percus-Yevick equation study is therefore not about critical exponents, but rather concerning the above discussed scenario of an exponentially decreasing pair correlation function with a correlation length approaching the radius of curvature $\kappa^{-1}$ from below. A clear confirmation of the scenario can be seen in Fig. 7 where both $h(r)$ and $\exp (\kappa r) h(r)$ are plotted in the vicinity of the critical point. Note 


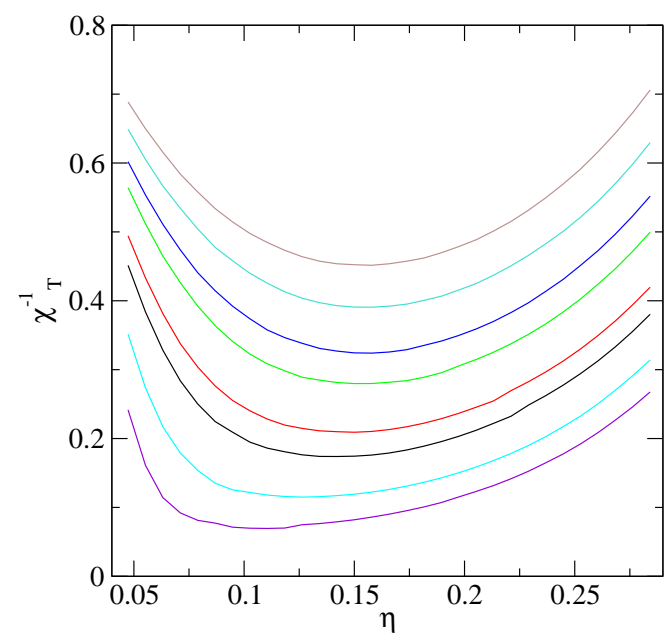

FIG. 6: Inverse of the isothermal compressibility versus packing fraction $\eta$ for the truncated Lennard-Jones fluid in $H_{2}$ within the PY approximation near but above the critical temperature: from top to bottom , $T=0.640,0.610,0.580,0.560,0.530,0.515,0.490,0.472$ (in the usual reduced units). The curvature parameter is $\kappa \sigma=0.5$.

that the gas-liquid critical point for $\kappa \sigma=0.5$ is found at a lower temperature than that in the Euclidean plane.

An interesting question concerning the critical point in the presence of negative curvature is whether the location of the point in the $(T, \rho)$ diagram goes continuously to the value in the Euclidean plane or goes to a lower, possibly zero-temperature point as the curvature is reduced to zero. The former possibility is natural if one thinks of the curvature as introducing a mere finite-size cut-off. However, the latter has been conjectured by Angles d'Auriac et al[95] in their study of the Ising model on hyperbolic lattices; these authors have also suggested the existence of a crossover line, emanating from the critical point in Euclidean space and extending to small nonzero curvature below which the pair correlation function has an algebraic decay for $r<\kappa^{-1}$ and an exponential decay at long distance. Approximate integral equations presumably predict a continuous behavior, and no crossover is seen around the location of the flat space critical point, but this may come from their intrinsic limitation. We have undertaken preliminary Molecular Dynamics simulations of the trun- 

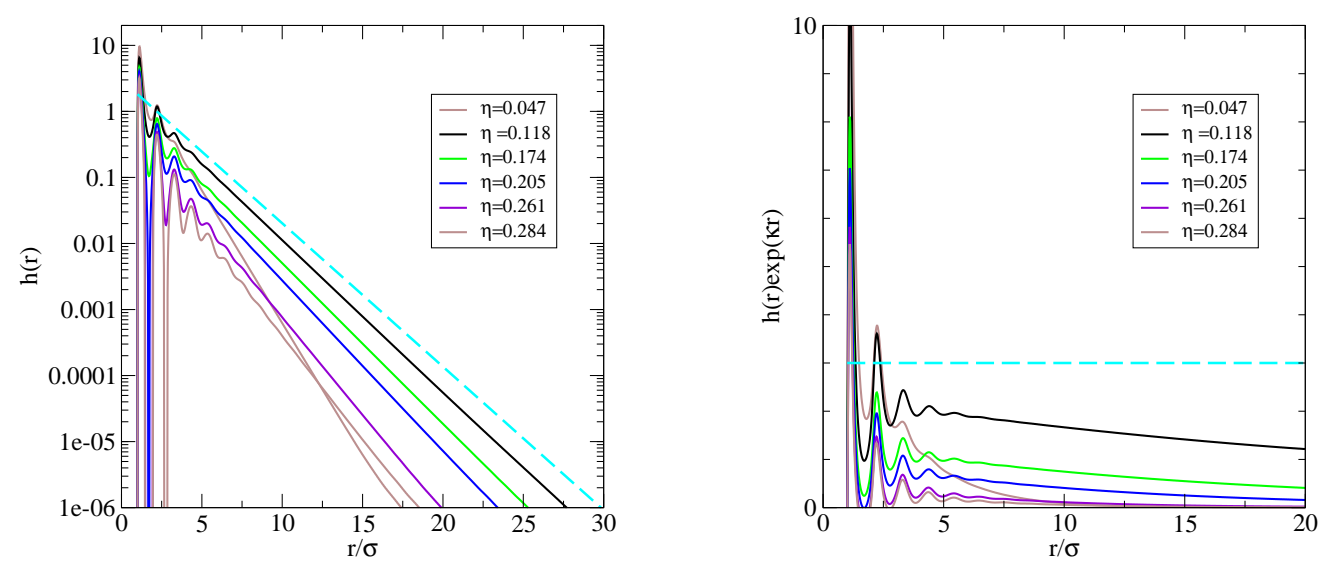

FIG. 7: (a) Log-linear plot of the pair correlation function $h(r)=g(r)-1$ of the truncated Lennard-Jones fluid in $\mathrm{H}_{2}$, as obtained in the PY approximation for $T=0.472$. The behavior as a function of $\eta$ is nonmonotonic and the slowest decay is for $\eta=0.118$, which corresponds to the maximum of the compressibility. The dotted line is $\exp (-\kappa r)$. (b) Same data multiplied by $\exp (\kappa r)$ : a convergence towards a constant plateau at large $r$ is clearly visible as one approaches the critical point. The curvature parameter is $\kappa \sigma=0.5$.

cated Lennard-Jones model in $\mathrm{H}_{2}$ to try to locate its gas-liquid critical point and possible crossover behavior. Fig. 8 displays a three-dimensional plot of the integral over space of $h(r)$ in the $(T, \phi)$ plane for a curvature parameter $\kappa \sigma=0.2$. Periodic boundary conditions with a fundamental polygon of 14 edges (see Appendix B and Ref.[34]) are used and the system size is unfortunately rather small (from 8 to 320 atoms). One observes that the integral indeed starts to rise very steeply as one reaches the vicinity of the Euclidean-space critical point and saturates at lower $T$ and $\rho$ due to system-size limitations. However, without studying finite-size effects by changing the fundamental polygon of the periodic boundary conditions, no clear-cut conclusion can be reached. Further work is needed to elucidate this question. 


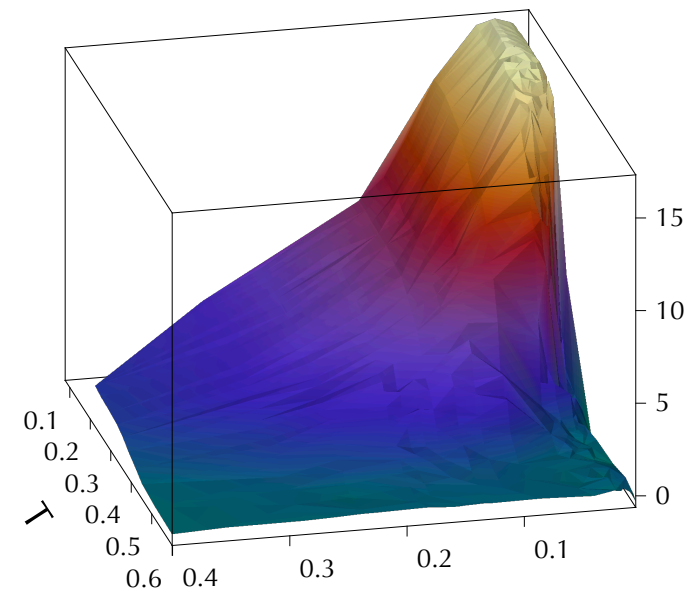

$\eta$

FIG. 8: MD simulation of the critical behavior of the truncated Lennard-Jones model in $H_{2}$ for $\kappa \sigma=0.2$. Three-dimensional plot of the integral of $h(r)$ over the system size as a function of $T$ and $\eta$. The critical point of the model in $E_{2}$ obtained by Monte Carlo simulation[97] is around $T_{c}=0.472$ and $\eta=0.33$.

\section{FREEZING, JAMMING AND THE GLASS TRANSITION}

\section{A. Geometric frustration}

Starting with the work of Frank[8], Bernal[98] and others in the fifties, a whole line of research has developed, trying to understand liquids, glasses and amorphous packings, i.e. systems with no apparent structural long-range order, from a geometric point of view. An explanation for the avoidance of crystal formation when cooling a liquid, with the resulting glass formation, as well as for the structure of glasses and amorphous materials has been put forward in terms of "geometric frustration" [7]. The latter describes a competition between a short-range tendency for the extension of a locally preferred order and global constraints that preclude tiling of the whole space by a periodic repetition of the local structure. A prototypical and well documented example is that of icosahedral order in three-dimensional systems in which particles interact through spherically symmetric pair potentials: despite being locally more favorable, icosahedral order built from tetrahedral units cannot freely propagate in space to give rise to long-range crystalline order. Icosahedral order therefore 
must come with topological defects. A step forward in the geometric description has been provided by the "curved space approach" developed by Kléman, Sadoc, Mosseri on the one hand [7, 10] and by Nelson, Sethna, and coworkers on the other [9, 99]. In the late seventies, Kleman and Sadoc[10] realized that perfect icosahedra could tile three-dimensional space if the metric of the latter were modified to introduce a constant positive curvature. On the hypersphere $S_{3}$ with a radius $R$ equal to the golden number $\left(\frac{1+\sqrt{5}}{2}\right)$ times the particle size, 120 particles form a perfect icosahedral tiling known as the $\{3,3,5\}$ polytope[7]. Such an unfrustrated crystallization is then expected to take place more easily, i.e. at higher temperature, than crystallization in Euclidean space which, due to frustration of icosahedral order, must involve a reorganization of the local order and a different, hexagonal closepacked or face-centered cubic, long-range order [57, 58]. The template or "ideal order" can then be used to describe real physical systems in Euclidean space, mostly metallic glasses, provided that one can described how it evolves when "flattening" space: roughly speaking, one observes in Euclidean space the remains of ideal icosahedral order that is broken up by the necessary appearance of topological defects, essentially disclination lines, which in some sense carry the curvature needed for compensating the flattening of the template. The same approach can be taken for a variety of local orders that are frustrated in Euclidean space but can tile space in curved spherical or hyperbolic geometries[7]. A theory of the glass transition has also been developed along these lines[100, 101].

Quite generally, curvature can be used as a tool to either frustrate or generate long-range order. In the above discussed example of icosahedral order, curvature allows one to build a perfect tetrahedral/icosahedral tiling in the form of a polytope in $S_{3}$. Generically, such polytopes can be found for special values of the radius of curvature expressed in units of the polytope edge: there are a finite number of them in spherical geometries and an infinite number in hyperbolic geometries (one could also consider geometries with spatially varying curvature)

Consider now two-dimensional space and fluids of particles interacting via spherically symmetric pair potentials. Such systems of disks on the Euclidean plane are not subject to geometric frustration: the locally preferred structure is a regular hexagon, with one atom at the center and 6 neighbors at the vertices, and this structure can be periodically repeated in space to form a triangular lattice. The system crystallizes extremely easily, either through a sequence of two continuous transitions with an intermediate hexatic phase 
as in the KTNHY [9, 80, 81] or through a weak first-order transition, and glass formation never occurs. Curving space then frustrates hexagonal order and forces in topological defects which are point-like in two dimensions. (The topic of hexagonal order and associated defects will be considered in more detail in Sec. VII.) Again, for specific values of the radius of curvature compared to the particle size, the spherical and hyperbolic manifolds ( $S_{2}$ and $H_{2}$, respectively) allow crystalline-like tilings which are conventionally represented by the Schläfli notation $\{p, q\}$ with $q$ the number of edges of the elementary polygonal tile and $p$ the number of polygons meeting at each vertex. Such $\{p, q\}$ tilings satisfy $(p-2)(q-2)=4$ in the Euclidean space $E_{2},(p-2)(q-2)<4$ on $S_{2}$ and $(p-2)(q-2)>4$ on $H_{2}$. This leaves the two dual triangular/hexagonal tilings $\{3,6\}$ and $\{6,3\}$ and the square tiling $\{4,4\}$ in $E_{2}$, the five tilings $\{3,3\},\{4,3\},\{3,4\},\{5,3\}$ and $\{3,5\}$, corresponding to the platonic solids, in $S_{2}$ and an infinity of tilings in $H_{2}[30]$.

The flexibility offered by the multiple tilings in $H_{2}$ has for instance been used by Modes and Kamien [31, 32] to study "isostatic" packings of hard disks in $H_{2}$. Isostaticity means that the number of constraints coming from force and torque balance equations is exactly equal to the number of degrees of freedom in the system[102]. It is a global requirement that is related to marginal stability in solids[103, 104]; it has recently received renewed attention in the context of "jamming phenomena" present in equilibrium and driven disordered assemblies of particles[105]. Isostaticity is a topological property that does not depend on the curvature of space. For a two-dimensional system of $N$ hard disks, there are $2 N$ degrees of freedom and $\bar{z} N / 2$ constraints coming form the number of contacts, where $\bar{z}$ is the average number of contacts per disk, so that isostaticity corresponds to $\bar{z}=4$. Therefore, all allowed tilings $\{4, q\}$ in $H_{2}$ are isostatic, which broadens the scope of systems that can be used to study the generic features associated with isostaticity [31, 32].

As stressed several times in this article, only hyperbolic geometry allows one to study macroscopic systems in the thermodynamic limit at constant nonzero curvature. Spherical geometry implies a finite system which can be investigated per se, as in studies of colloidal systems in spherical substrates, or used as trick to converge to the thermodynamic limit in the Euclidean plane by decreasing the curvature. The latter approach, corresponding to the already mentioned spherical boundary conditions, has been taken to study dense disordered packings of hard particles [59, 71] as well as the nature of crystallization for particles interacting with power-law and logarithmic pair potentials [65, 67]. In both cases, the rationale 
is that spherical boundary conditions do not favor crystalline hexagonal arrangements as much as periodic boundary conditions directly implemented in $E_{2}$, which then provides a less biased finite-size approach to phenomena taking place in flat space in the thermodynamic limit. On the other hand, one may be interested in studying the influence of geometric frustration on the structure and the dynamics of a system and therefore work with a constant nonzero curvature. Consider the hyperbolic plane $\mathrm{H}_{2}$ which, as already stated, is of infinite spatial extent. For disks of diameter $\sigma$ embedded in $H_{2}$, the local order of the liquid changes as one increases the radius of curvature $\kappa^{-1}$. From hexagonal at zero and small curvature parameter $\kappa \sigma$, it becomes heptagonal at a larger $\kappa \sigma$, then octogonal, etc[1], and for commensurate values of the curvature parameter, the locally preferred structure can freely propagate to tile space; in such cases, corresponding to[11]

$$
\kappa_{n} \sigma=2 \cosh ^{-1}\left[\frac{1}{2 \sin \left(\frac{\pi}{n}\right)}\right]
$$

with $n=6,7,8, \ldots$, there is no frustration. The effect of frustration can be investigated off these commensurate curvatures. For instance, frustrated hexagonal order can be studied for $\kappa \sigma$ larger than zero $\left(\kappa_{6} \sigma=0\right)$ but significantly less than $\kappa_{7} \sigma \simeq 1.09055[11]$.

Before moving on to a more extensive discussion of frustration and glass formation in a liquid model in the hyperbolic plane, we briefly describe the various tools that have been used in practice to assess the structure of dense phases in curved space. All of them are actually extensions of methods and observables developed for the Euclidean case. For spherical particles, both "positional" (translational) and "bond-orientational" orders are of interest. "Positional" refers to the distribution functions introduced in Sec. [V] that involve correlations between the particle centers. The most easily measured or computed is the radial distribution function $g(r)$, with $r$ being the geodesic distance between two particle centers. "Bond-orientational" refers to the distribution functions associated with the (artificial) "bonds" joining two nearest-neighbor particles. There are different ways of defining such bonds, a commonly used one being to assign nearest neighbors through a Voronoi-Dirichlet construction (see below). In Euclidean space, one associates a unit vector to each bond and orientational order refers to the average and the correlations of local order parameters defined in two dimensions as 9 


$$
\Psi_{n}\left(\mathbf{r}_{j}\right)=\frac{1}{N_{b}} \sum_{k=1}^{N_{b}} \exp \left(i n \theta_{j k}\right),
$$

where the sum is over the $N_{b}$ nearest neighbors of the particle located at $\mathbf{r}_{j}$ and $\theta_{j k}$ is the angle characterizing the "bond" between atoms $j$ and $k(n=6$ for instance is characteristic of 6 -fold hexagonal or hexatic order). Similar quantities can be introduced in higher dimensions [106].

The difficulty that one faces when dealing with non-Euclidean space is that there is no global existence of vector fields: vectors are defined locally (in the tangent Euclidean manifold) and to be combined or compared to vectors at another point in space, they must be "parallel transported" along the geodesic joining the points (see Appendix A and Refs. [7, 38]). As a result, the bond-orientational correlation functions involving an extension to non-Euclidean geometry of the local order parameters in Eq. 6.2 are path-dependent in the presence of a nonzero curvature. A natural definition of the pair correlation functions is however to consider the geodesic between the two points under consideration, which leads to

$$
g_{n}(r)=\frac{1}{N} \sum_{i, j=1}^{n}\left\langle\tilde{\Psi}_{n}(i \mid j) \Psi_{n}^{*}(j)\right\rangle_{\Gamma_{i j}} \delta^{(2)}\left(r_{i j}-r\right),
$$

where $\tilde{\Psi}_{n}(i \mid j)$ is the order parameter when parallel transported from point $\mathbf{r}_{i}$ to point $\mathbf{r}_{j}$ along the geodesic $\Gamma_{i j}, r_{i j}$ is the geodesic distance between the two points and $\delta^{(2)}$ is the delta function that is appropriate for the non-Euclidean metric (for $S_{2}$, see Ref. 60] and for $\mathrm{H}_{2}$ see Refs.[11, 69]). Finally, the Voronoi tesselation or its dual, the Delaunay construction (or Dirichlet triangulation in two dimensions), is a way to uniquely define nearest neighbors, hence bonds, coordination number, etc, which is most useful in dense liquid and amorphous phases. In particular, it allows one to characterize at a microscopic level topological defects (especially disclinations which are orientational defects in the form of lines in three dimensions and points in two dimensions) occurring in a putative order. These constructions can be extended to spherical[7, [57, 60] and hyperbolic geometry [11, 70, 107].

\section{B. Glassforming liquid on the hyperbolic plane}

We briefly review here the extensive computer simulation study that we have recently carried out on the structure and the dynamics of the truncated Lennard-Jones liquid (see 


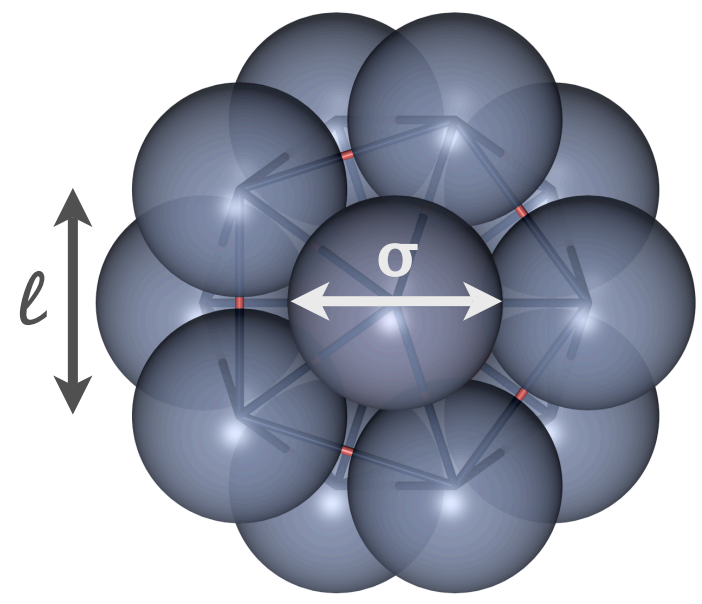

(a)

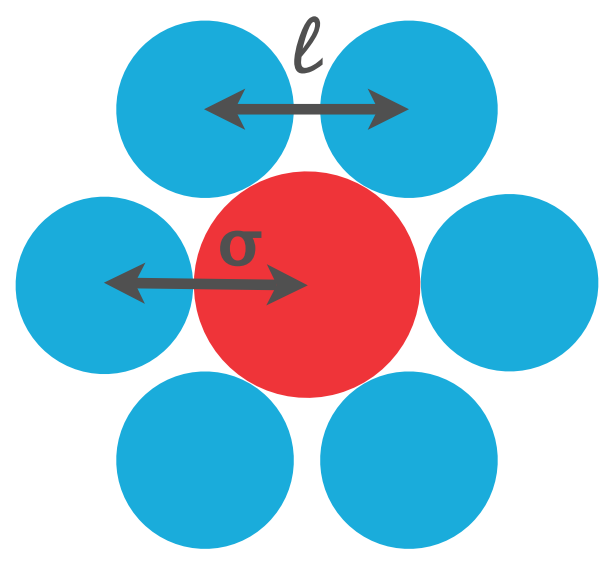

(b)

FIG. 9: Locally preferred structure in atomic liquids: (a) Frustrated icosahedral order in three-dimensional Euclidean space $E_{3}$ : the distance $l$ between two neighboring outer spheres is slightly larger than the particle diameter $\sigma$ which is also the distance between the central sphere and the 12 outer ones. (b) Frustrated hexagonal order in the hyperbolic plane $H^{2}$ : the nonzero curvature induces that, here too, $l$ is slightly larger than $\sigma$.

above) embedded in $H_{2}$ with curvature parameter $\kappa \sigma$ small enough that the local order in the liquid is hexagonal/hexatic and is therefore frustrated at long distance. The motivation behind this study was to assess the validity of the frustration-based theory of the glass transition [100, 101]. In the latter, frustration is hypothesized to be ubiquitous in liquids. The salient features of the phenomenology of glassforming liquids, above all the spectacular "super-Arrhenius" increase of the viscosity and the relaxation time as one lowers the temperature[101, are then attributed to the frustration-limited extension of the locally preferred liquid order; this extension is driven by the proximity to an "avoided ordering transition" that would take place in the absence of frustration[100, 101]. Frustrated icosahedral order in three dimensions with $S_{3}$ as the unfrustrated space [7, 9] is one example that we have already discussed. A simpler model however is provided by frustrated hexagonal order in two-dimensional negatively curved space [9, 11, 12], as illustrated in Fig. 9. In this case, "ideal ordering" takes place in flat space and this ordering transition observed at a temperature $T^{*}$ is avoided as soon as one introduces a nonzero curvature, with the curvature parameter $\kappa \sigma$ playing the role of the frustration strength. 


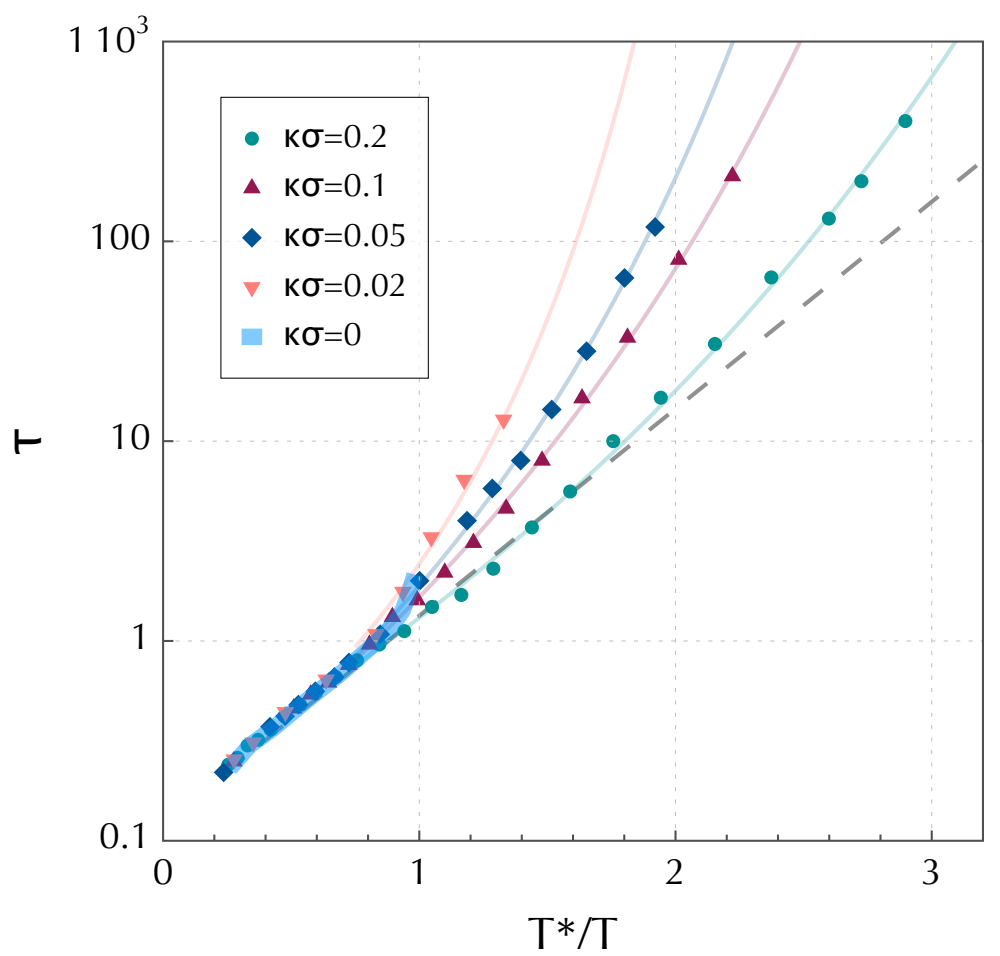

FIG. 10: Slowing down of relaxation in the truncated Lennard-Jones model in $H_{2}$ (MD simulation [68]): logarithm of the translational relaxation time $\tau$ versus $T^{*} / T$ for $\rho \simeq 0.85$ and for various curvature parameters $\kappa \sigma\left(T^{*}\right.$ is the ordering temperature in $\left.E_{2}\right)$. The dotted line is the Arrhenius $T$-dependence roughly observed at temperatures above $T^{*}$. When $\kappa \sigma>0$, the system remains liquid below $T^{*}$ until it forms a glass. The deviation from Arrhenius behavior increases when curvature (hence frustration) decreases.

Glass formation which is preempted by ordering at $T^{*}$ in the Euclidean plane becomes possible in the hyperbolic plane and the liquid phase can be kept in equilibrium at temperatures below $T^{*}$. This is shown in Fig. 10 where we plot the logarithm of the relaxation time versus $1 / T$ for several curvatures, as obtained from Molecular Dynamics simulation. (Details on the Molecular Dynamics simulation technique in hyperbolic geometry and on the generalization of the time-dependent correlation functions are given in Refs. [68 70].) One observes that curvature plays virtually no role for temperatures above $T^{*}$ and that super-Arrhenius behavior, i.e. a deviation from simple Arrhenius $T$-dependence, becomes significant around $T^{*}$ and is more pronounced as one decreases the curvature parameter $\kappa \sigma$. These observations support the theoretical predictions that the avoided transition controls the slowing down of the relaxation and that the "fragility " of a glassformer, which quantifies 
how much it deviates from simple Arrhenius behavior, decreases as one increases the frustration, i.e here the curvature. Note that this trend should also apply to liquids of colloidal particles on a spherical substrate: at least for small enough curvature such that the local order remains hexagonal, decreasing the curvature should lead to a stronger slowing down of the dynamics.

The effect of the curvature can also be seen on the structure of the liquid. This is illustrated in Fig. 11 where the radial distribution function $g(r)$ of the Lennard-Jones model in the Euclidean plane is compared to that in $H_{2}$ with $\kappa \sigma=0.1$. The (quasi) long-range order present below $T^{*}$ in flat (unfrustrated) space is no longer observed in curved space, thereby confirming that the one-component system remains a disordered liquid in the latter.

On general grounds, one expects that curvature-generated frustration induces three different regimes in a liquid as temperature decreases. In a first regime, above $T^{*}$, the structure and the dynamics only involve local properties; as, locally, curved space looks flat, there is no significant influence of curvature (at least for small curvatures that accommodate the same local order). A second regime near and below $T^{*}$ is controlled by the proximity of the avoided transition: the locally preferred structure extends in space and the associated correlation length grows with decreasing temperature (as can be checked by studying the bondorientational order correlation function $G_{6}(r)=g_{6}(r) / g(r)$, see Ref.[69]). A final regime is reached when the correlation length associated with growing (bond-orientational) order saturates due to frustration: the spatial extent of the frustrated order cannot grow beyond the radius of curvature and the final regime is dominated by the presence of an irreducible density of topological defects. More will be said below about this regime. This three-regime scenario has been observed in computer simulations of liquids in both negative [69, 70, and positive 60] curvature manifolds.

\section{GROUND-STATE PROPERTIES, ORDER AND DEFECTS}

So far, we have mostly dealt with liquid and fluid phases in curved space. We briefly discuss now low-temperature (or high-density) phases and ground-state properties. In the preceding section, we have stressed that a nonzero curvature can either induce long-range

order[108], allowing tiling of a homogeneous curved space by regular polytopes for specific values of the curvature, or, on the contrary, frustrate the long-range order present in Eu- 


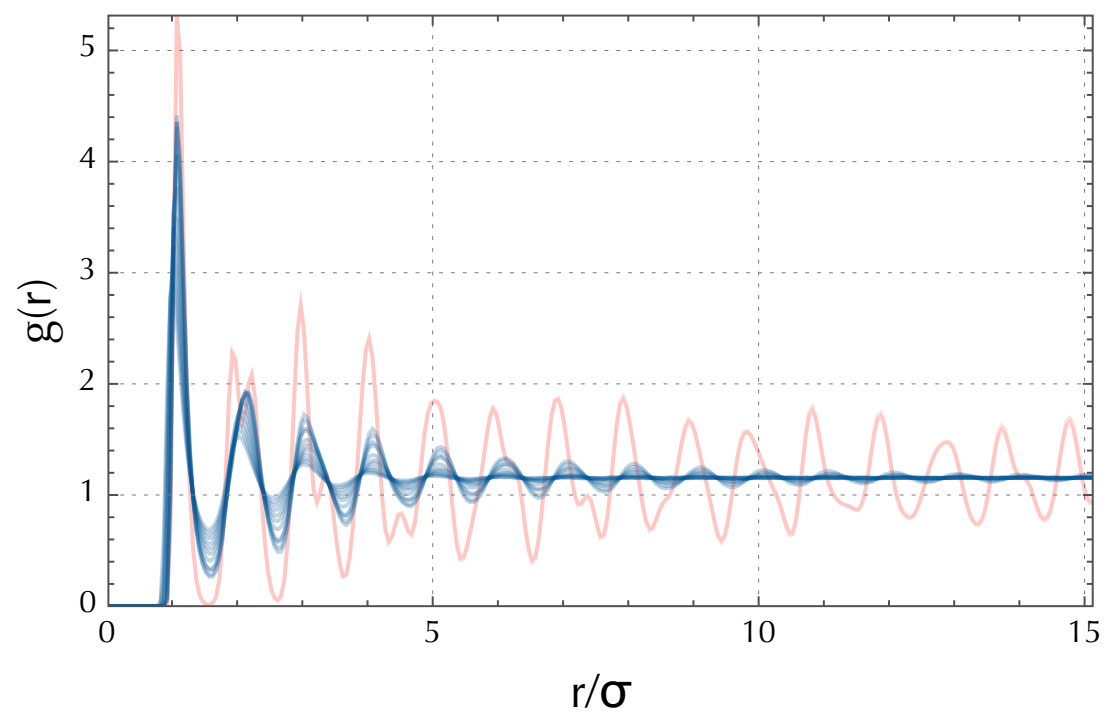

(a)

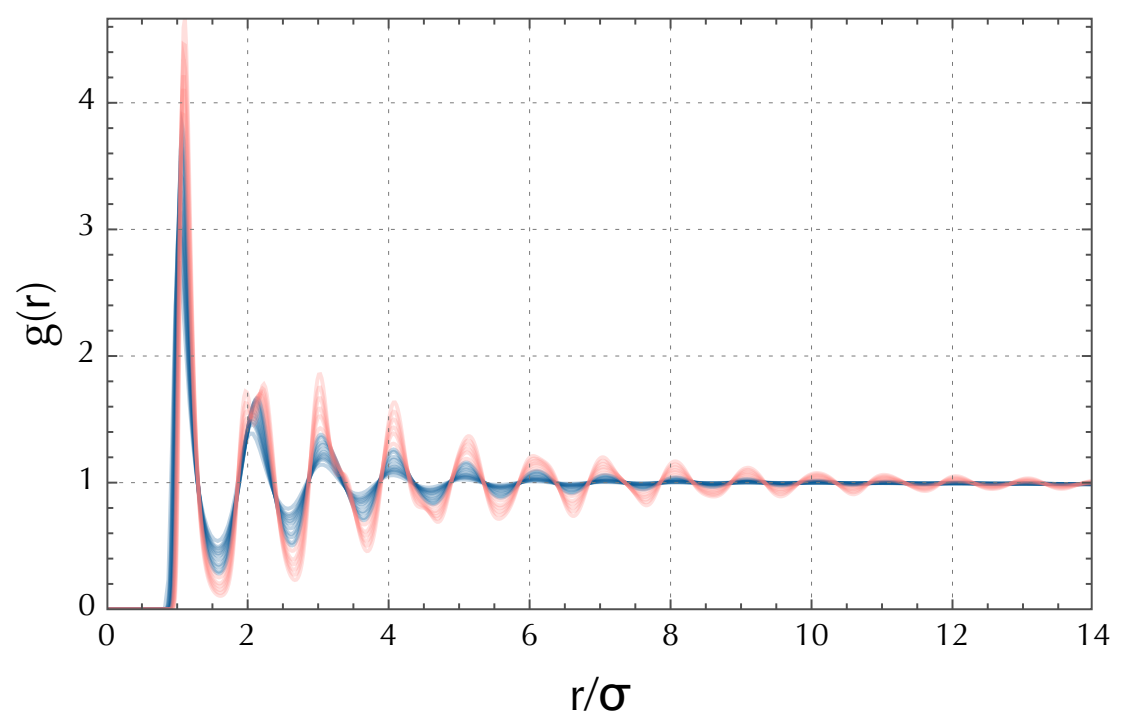

(b)

FIG. 11: Radial distribution function of the truncated Lennard-Jones model in $H_{2}$. The (blue) curves correspond to temperatures above the flat-space ordering transition $\left(T>T^{*}\right)$. (a) Euclidean plane $(\kappa \sigma=0)$ : when $T<T^{*}$ (red curves) $g(r)$ displays many peaks corresponding to the existence of a quasi-long range positional order. (b) Hyperbolic plane with $\kappa \sigma=0.1$ : when $T<T^{*}$ (red curves), there is no signature of quasi-long range order and the system remains in a liquid phase. 
clidean space. We focus on the latter situation and we moreover restrict the discussion to the two-dimensional case with spherical particles, in which the hexagonal/triangular order that forms the ground-state in flat space is frustrated by the introduction of curvature. The topic of order, curvature and defects in two dimensions has recently been nicely reviewed in a quite exhaustive article by Bowick and Giomi[27]. Therefore, we only intend to give a brief survey of this aspect, for the sake of completeness of this article.

A first insight into frustrated hexagonal order in non-Euclidean geometries is provided by topological considerations. Consider the Delaunay triangulation of a dense assembly of particles on a two-dimensional manifold of genus $g$ (see Sec. VI) and, if open, with $h$ boundaries. Its Euler characteristic is then $\chi=2(1-g)$ and the Euler-Poincaré theorem states that any "tesselation" (i.e. tiling) of the manifold satisfies

$$
V-E+F=\chi,
$$

where $V, E$ and $F$ are the numbers of vertices, edges and faces in the tesselation. In the case of the Delaunay tesselation by triangles, this leads to

$$
\frac{N}{6}(6-\bar{z})=\chi,
$$

where $N \equiv V$ is the number of particle centers (i.e., of vertices) and $\bar{z}$ is the average coordination number of the particles. If one defines the topological charge $q_{i}$ of a particle with coordination number $z_{i}$ as $q_{i}=6-z_{i}$, one can rewrite Eq. 7.2 as

$$
\sum_{i=1}^{N} q_{i}=6 \chi=12(1-g)-6 h .
$$

For the Euclidean plane, $g=1$ and $h=0$, so that $\chi=0$ and $\bar{z}=6$ : perfect hexagonal tiling is possible. For the sphere, $S_{2}, g=2$ and $h=0$, so that the average coordination number must be strictly less than 6 . The minimal way to satisfy Eq. 7.3 is then to have 12 particles with configuration number number 5 in an otherwise 6 -fold coordinated configuration. Such particles appear as (point) topological defects in hexagonal order. They represent positively charged $(q=+1)$ disclinations. For the hyperbolic plane $H_{2}$, one may first take a detour via periodic boundary conditions (see Appendix B). When imposing a periodic boundary condition, the primitive cell containing the system corresponds to a (compact) quotient space of genus $g \geq 2$, hence with $\chi=-2(g-1)$. In consequence, the total topological charge in the primitive cell has to be equal to $-12(g-1)$ : there must be an excess of negatively 
charged disclinations in this case (e.g. of disclinations with $q=-1$ corresponding to 7-fold coordinated particles). By using geometric input in the form of the Gauss-Bonnet theorem that relates curvature $K=-\kappa^{2}$ and characteristic $\chi$,

$$
\int_{\Sigma} d S K=2 \pi \chi
$$

where we recall that $d S=\sqrt{|g(x)|} d^{2} x$ (see Eq. 4.2 ) and $\Sigma$ is the primitive cell (or fundamental polygon) of the periodic boundary condition, one finds that the area of the primitive cell is given by $A=-2 \pi \chi \kappa^{-2}$. As a result, Eq. (7.3) becomes

$$
\frac{1}{A} \sum_{i=1}^{N} q_{i}=-\left(\frac{3}{\pi}\right) \kappa^{2}
$$

which means that the density of topological charge in $H_{2}$ is only controlled by the curvature, irrespective of the choice of periodic boundary condition, thereby providing a well defined thermodynamic limit for a "bulk" property (see also the discussion in Secs. III and IV).

In curved two-dimensional space, hexagonal order must come with an irreductible number (or density) of topological defects even in the ground state. Actually, constructing the ground state of a system of particles on a two-dimensional manifold is highly nontrivial. The question goes back to Thomson who considered the ground state of repulsive charged particles on a sphere[109]. (Another famous example is the explanation by Caspar and Klug[110] of the icosahedral symmetry of spherical virus capsids.) Brute-force numerical approaches are plagued by the presence of multiple low-energy minima, which are induced by geometric frustration, and finding the global minimum for a large number of particles is extremely difficult. To get around this problem, coarse-grained approaches have been devised, most prominently a continuum elastic theory developed by Nelson, Bowick, Travesset and their coworkers [27, 111 116]. In the latter, one directly deals with the defect degrees of freedom, and all the microscopic information about particle interactions is embedded in effective elastic constants and defect core energies[27]. (Note that an alternative route to the continuum elastic theory has recently been proposed on the basis of a coarse-grained density functional theory: see Ref.[117].)

In the Bowick-Nelson-Travesset approach[112], one focuses on the density of disclinations, which are the elementary defects from which other defects such as dislocations can be built, and treats the 6 -fold coordinated particles through continuum elastic theory. At low 
temperature, the elastic free energy of an arbitrary disclination density

$$
s(\mathbf{x})=\frac{\pi}{3 \sqrt{|g(\mathbf{x})|}} \sum_{i=1} q_{i} \delta^{(2)}\left(\mathbf{x}-\mathbf{x}_{i}\right)
$$

where $N_{d}$ is the total number of disclinations, is given by [27, 112$]$

$$
F[s]=\frac{Y}{2} \int_{\Sigma} \int_{\Sigma} d^{2} x \sqrt{|g(\mathbf{x})|} d^{2} y \sqrt{|g(\mathbf{y})|}(s(\mathbf{x})-K(\mathbf{x})) G_{2}(\mathbf{x}, \mathbf{y})(s(\mathbf{y})-K(\mathbf{y}))+\sum_{i=1}^{N_{d}} E_{c o r e, i}
$$

where $Y$ is the Young modulus of the hexagonal crystal in flat space, $E_{\text {core }, i}$ is a disclinationcore free energy renormalized by thermal fluctuations [112], $K(\mathbf{x})$ is the Gaussian curvature at point $\mathbf{x}$, and $G_{2}(\mathbf{x}, \mathbf{y})$ is the Green function of the bi-Laplacian (or biharmonic operator) on the manifold $\Sigma$, i.e. satisfying

$$
\Delta^{2} G_{2}(\mathbf{x})=\delta^{(2)}(\mathbf{x})
$$

with $\Delta$ the Laplace-Beltrami operator. The first term of the free-energy functional is minimized by having $s(\mathbf{x})=K(\mathbf{x})$ at each point, which means that the distribution density exactly cancels the effect of the Gaussian curvature. If one considers homogeneous manifolds of constant Gaussian curvature, $K(\mathbf{x}) \equiv K$, such as $S_{2}$ and $H_{2}$, the former condition cannot be everywhere satisfied as the disclinations are discrete objects. Topology however requires a generalization of the electroneutrality constraint, namely

$$
\int_{\Sigma} d^{2} x \sqrt{|g(\mathbf{x})|}(s(\mathbf{x})-K)=0
$$

which by inserting Eq. (7.6) and the Gauss-Bonnet theorem, Eq. (7.4), is equivalent to Eq. (7.6). Therefore, on average, the disclination charges screen the Gaussian curvature.

As the core energy of the dislocations increases with the magnitude of their topological charge, the second term of the free-energy functional is minimized by having the smallest irreducible number of disclinations with elementary topological charges, e.g. 12 disclinations of charge $q=+1$ on $S_{2}$ and a density of disclinations of charges $q=-1$ equal to $\frac{3}{\pi} \kappa^{2}$ on $H_{2}$.

For small enough curvature (which for $S_{2}$ means a large enough number of particles if the particle size $\sigma$ is kept fixed), adding extra disclinations in the system on top of the irreducible number may lower the (free) energy by screening more efficiently the curvature [27, 112, 118]. The total charge of these extra disclinations is zero (so that Eq. (7.3) remains satisfied) and the most economical way for them to organize is by forming dislocations that consist of 


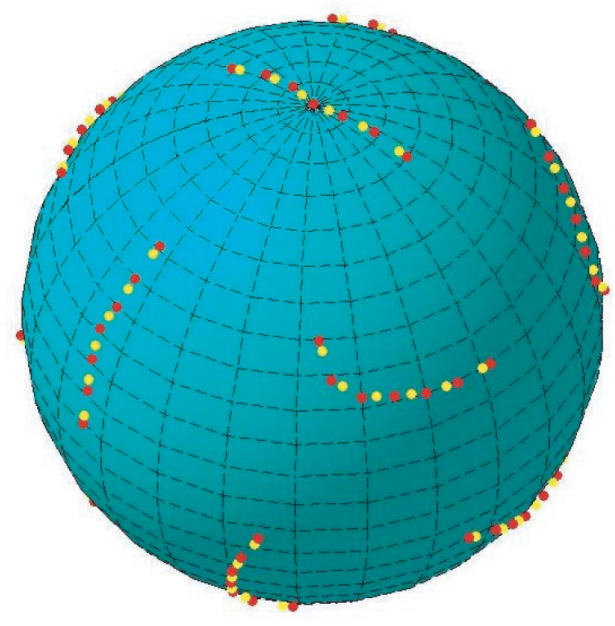

(a)

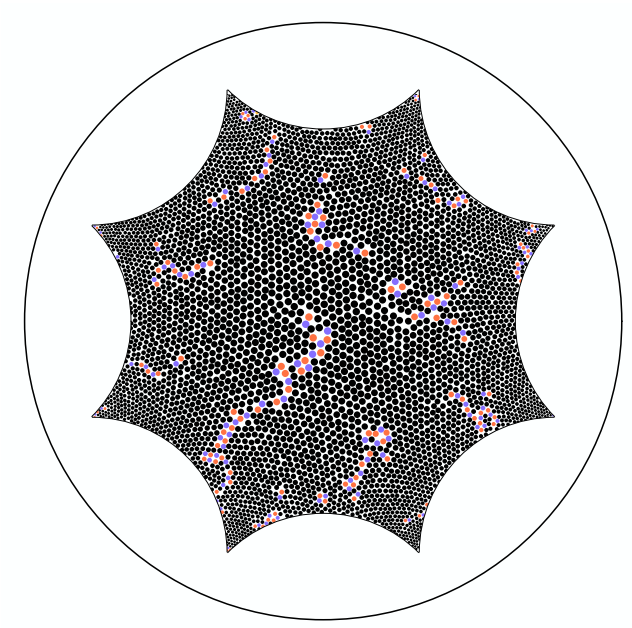

(b)

FIG. 12: Illustration of grain boundary scars in a hexagonal background in two-dimensional curved space. (a) Model grain boundaries in $S_{2}$ obtained by minimization of Eq. (7.7) [18]. (b) Low-temperature atomic configurations of the truncated Lennard-Jones liquid in the Poincaré disk representation of $H^{2}$ [69] (see Appendix A for the definition of Poincaré disk).

"dipoles" made by a positive and a negative disclination. The surprising outcome of the theoretical studies on order on the sphere $S_{2}$ is that these dislocations form strings that radiate from each of the irreductible disclinations and terminate in the hexagonal crystalline background at a finite distance[27, 112, 118]. These structures which are found found on the hyperbolic plane[70], but are forbidden in Euclidean space, have been dubbed "grain boundary scars "[18, 112]. We illustrate these structures on $S_{2}[18]$ and $H_{2}[70]$ in Fig. 12 . The theory predicts the number of dislocations per irreductible disclination as well as the cut-off distance $r_{c}$ before which the strings of dislocations terminate. For instance, the latter is given in $S_{2}$ by 112$]$

$$
r_{c}=R \arccos \left(\frac{5}{6}\right) \simeq 0.59 R
$$

and in $H_{2}$ by $[70]$

$$
r_{c}=\kappa^{-1} \operatorname{arccosh}\left(\frac{7}{6}\right) \simeq 0.57 R
$$

regardless of the microscopic details of the system of particles. These predictions concerning 
grain boundary scars in $S_{2}$ and $H_{2}$ are well supported by experimental[18, 119] and computer simulation results[27, 70, 117]. The elastic theory based on defects has been generalized to long range interactions between particles on a sphere, to other two-dimensional manifolds, with varying curvature and possibly open, and to other type of order: this is discussed in detail in the review of Bowick and Giomi[27], where all relevant references can also be found. In addition, the dynamics of the defects, essentially the diffusion of the dislocations, has been studied theoretically and experimentally on the sphere[17, 27, 113] as well as theoretically and by computer simulation in the hyperbolic plane[70].

\section{CONCLUSION}

In this article, we have reviewed the work on the structure and the dynamics of fluids, liquids, and more generally dense phases, in curved space. We have stressed that the motivation for such studies is twofold. On the one hand, curving space provides an additional control parameter, curvature, to shed light on the behavior of systems of interest in the flat, Euclidean space. Examples are provided by the use of spherical and hyperspherical boundary conditions as well as by investigations of the glass transition in the context of geometric frustration. On the other hand, there are many physical situations in physical chemistry, soft condensed matter and material science in which a two-dimensional curved surface is coated by a layer of particles that can move and equilibrate on the curved substrate. We have focused on cases where the geometry of the substrate is frozen, and most theoretical developments have been presented for the case of homogeneous two-dimensional manifolds of constant Gaussian curvature, the sphere $S_{2}$ and the hyperbolic plane $\mathrm{H}_{2}$.

Non-Euclidean geometries bring in subtleties in the statistical mechanics of systems of particles when compared to the standard Euclidean case. We have discussed the main ones, concerning the thermodynamic limit and the effect of the boundaries, the definition of the pressure and the relations involving correlation functions, the constraints on the form of the interaction potentials, the properties of particle diffusion, or the nature and the organization of the defects in dense and quasi-ordered phases. Additional results can be summarized as follows: curvature has a negligible or weak effect on the fluid behavior at high temperature and/or low density when the local structure or dynamics are probed. On the other hand, a nonzero curvature has strong consequences in dense phases where it can either induce 
or frustrate ordering. In the latter case, it slows down the relaxation and allows glass formation, as well as imposes in an irreducible number of topological defects in ground-state and low-temperature configurations. Curvature has also a distinct influence when longrange interactions, as in Coulombic systems, or long-range correlations, as in the vicinity of a critical point, are expected: there, the radius of curvature acts as a cutoff that imposes exponential decay at long distances.

Needless to say that progress should be made to describe liquids and fluids on substrates with varying curvature or even with fluctuating curvature as encountered in membranes. Above all, one can hope that in the near future more experiments will be performed on curved substrates to provide systematic information on the phase behavior and the dynamics of particle systems for a significant range of temperature or density and for several curvatures or geometries.

\section{Appendix A: A recap on Riemannian manifolds}

To describe liquids on curved surfaces, let us introduce some elements of differential geometry [7, 37, 38]. A $d$-dimensional Riemannian manifold is defined by a set of coordinates $\mathbf{x}=\left(x_{1}, x_{2}, \ldots, x_{d}\right)$ and a metric tensor $g_{i j}(\mathbf{x})$. The length element $d s$ is defined by

$$
d s^{2}=\sum_{i j}^{d} g_{i j}(\mathbf{x}) d x_{i} d x_{j},
$$

and the "volume" element is given by

$$
d S=\sqrt{|g(\mathbf{x})|} \prod_{i=1}^{d} d x_{i},
$$

where $|g(\mathbf{x})|$ denotes the absolute value of the determinant of the metric tensor.

Diagonalizing the curvature tensor provides the principal directions of the manifold (eigenvectors) at point $\mathbf{x}$ and the eigenvalues are the radii of curvature, $R_{i}, i=1, \ldots, d$. The mean curvature is defined as the algebraic average of the curvatures,

$$
k(\mathbf{x})=\frac{1}{d} \sum_{i=1}^{d} \frac{1}{R_{i}(\mathbf{x})} .
$$

In the case where the manifold is two-dimensional, one defines the Gaussian curvature as the product of the two curvatures,

$$
K(\mathbf{x})=\frac{1}{R_{1}(\mathbf{x}) R_{2}(\mathbf{x})} .
$$


If $X$ is a vector field (defined at each point $\mathbf{x}$ in the tangent Euclidean manifold), the divergence is given by

$$
\operatorname{div}(X)=\frac{1}{\sqrt{|g(\mathbf{x})|}} \sum_{i=1}^{d} \frac{\partial\left(\sqrt{|g(\mathbf{x})|} X^{i}\right)}{\partial x_{i}}
$$

and the gradient of the scalar function $f$

$$
(\operatorname{grad}(f))^{i}=\sum_{j=1}^{d} g^{i j} \frac{\partial f}{\partial x_{j}}
$$

where $g^{i j}(\mathbf{x})$ is the inverse tensor of $g_{i j}(\mathbf{x})$, i.e. $\sum_{j=1}^{d} g_{i j} g^{j k}=\delta_{i}^{k}$ with $\delta_{i}^{k}$ the Kronecker symbol.

The Laplace-Beltrami operator $\Delta$ acts on a function $f(\mathbf{x})$ as $\Delta f=\operatorname{div} \operatorname{grad}(f)$, i.e.,

$$
\Delta f=\frac{1}{\sqrt{|g(\mathbf{x})|}} \sum_{i=1}^{d} \frac{\partial}{\partial x_{i}}\left(\sqrt{|g(\mathbf{x})|} g^{i j}(\mathbf{x}) \frac{\partial f}{\partial x_{j}}\right)
$$

We mostly consider in this article homogeneous manifolds of constant nonzero curvature. In two dimensions, there are two associated geometries, the spherical and the hyperbolic ones. For the sphere $S_{2}$, the metric tensor is diagonal in the angular coordinates $\theta$ (colatitude) and $\phi$ (longitude) and one has

$$
g_{\theta \theta}=R^{2}, \quad g_{\phi \phi}=R^{2} \sin (\theta)^{2} .
$$

The squared length element $d s^{2}$ is then equal to

$$
d s^{2}=R^{2}\left(d \theta^{2}+\sin (\theta)^{2} d \phi^{2}\right)
$$

the area element is given by

$$
d S=R^{2} \sin (\theta) d \phi d \theta
$$

and the two radii of curvature are equal and constant with $R_{1}=R_{2}=R$ and the Gaussian curvature $K=R^{-2}$. Finally, the Laplace-Beltrami operator is

$$
\Delta=\frac{1}{\sin (\theta)} \frac{\partial}{\partial \theta}\left(\sin (\theta) \frac{\partial}{\partial \theta}\right)+\left(\frac{1}{\sin (\theta)}\right)^{2} \frac{\partial^{2}}{\partial \phi^{2}} .
$$

For the hyperbolic plane $H_{2}$ (also called "pseudosphere" or "Bolyai-Lobachevski plane") [29, 30, one can use the polar coordinates $r$ and $\phi$. The metric tensor is then diagonal with $g_{r r}=1$ and $g_{\phi \phi}=\kappa^{-2} \sinh (\kappa r)^{2}$, which gives a squared length element

$$
d s^{2}=d r^{2}+\left(\frac{\sinh (\kappa r)}{\kappa}\right) d \phi^{2}
$$


and an area element

$$
d S=\frac{\sinh (\kappa r)}{\kappa} d r d \phi
$$

The two radii of curvature are of opposite signs, $R_{1}=-R_{2}=\kappa^{-1}$, so that $K=-\kappa^{2}$. In addition, the Laplace-Beltrami operator is given by

$$
\Delta=\frac{1}{\sinh (\kappa r)}\left(\frac{\partial}{\partial r} \sinh (\kappa r) \frac{\partial}{\partial r}\right)+\left(\frac{\kappa}{\sinh (\kappa r)}\right)^{2} \frac{\partial^{2}}{\partial \phi^{2}} .
$$

The hyperbolic plane cannot be embedded in three-dimensional Euclidean space (contrary to $S_{2}$ ) and "models", i.e. projections must be used for its visualization. A convenient one is the Poincaré disk model which projects the whole hyperbolic plane $H_{2}$ onto a unit disk. The projection is conformal (angles are conserved) but not isometric (distances are deformed and shrinks as one reaches the disk boundary). This representation is used in Fig. 12 b. If $x$ and $y$ are the Cartesian coordinates of a point on the unit disk, the relation to the above polar coordinate is as follows:

$$
\begin{aligned}
\sqrt{x^{2}+y^{2}} & =\tanh \left(\frac{\kappa r}{2}\right), \\
\frac{y}{x} & =\tan (\phi),
\end{aligned}
$$

and the squared length element $d s^{2}$ is given by

$$
\mathrm{d} s^{2}=\kappa^{-2} \frac{4\left(\mathrm{~d} x^{2}+\mathrm{d} y^{2}\right)}{\left(1-\left(x^{2}+y^{2}\right)\right)^{2}} .
$$

From the above formulae, one can compute for instance the geodesic distance $r_{12}$ between two points $\mathbf{r}_{1}$ and $\mathbf{r}_{2}$. In $S_{2}$, one finds

$$
\cos \left(\theta_{12}\right)=\cos \left(\theta_{1}\right) \cos \left(\theta_{2}\right)-\sin \left(\theta_{1}\right) \sin \left(\theta_{2}\right) \cos \left(\phi_{1}-\phi_{2}\right)
$$

where $\left(\theta_{1}, \phi_{1}\right)$ and $\left(\theta_{2}, \phi_{2}\right)$ are the coordinates of the two points and $R \theta_{12}$ the geodesic distance between these points. On the other hand in $H_{2}$, one has

$$
\cosh \left(\kappa r_{12}\right)=\cosh \left(\kappa r_{1}\right) \cosh \left(\kappa r_{2}\right)-\sinh \left(\kappa r_{1}\right) \sinh \left(\kappa r_{2}\right) \cos \left(\phi_{1}-\phi_{2}\right),
$$

where $\left(r_{1}, \phi_{1}\right)$ and $\left(r_{2}, \phi_{2}\right)$ are the (polar) coordinates of the two points. Note the symmetry between Eqs. A18 and A19 in the exchange of $R \leftrightarrow i \kappa^{-1}$. The same symmetry takes place in relating spherical and hyperbolic trigonometries. So for instance, from the standard 
result on a sphere, one finds the following trigonometric relations for the a general hyperbolic triangle with sides $a, b$, and $c$ and opposite angle $\alpha, \beta$ and $\gamma[30]$ :

$$
\begin{gathered}
\frac{\sinh (\kappa a)}{\sin (\alpha)}=\frac{\sinh (\kappa b)}{\sin (\beta)}=\frac{\sinh (\kappa c)}{\sin (\gamma)} \\
\cosh (\kappa c)=\cosh (\kappa a) \cosh (\kappa b)-\sinh (\kappa a) \sinh (\kappa b) \cos (\gamma), \\
\cosh (\kappa c)=\frac{\cos (\alpha) \cos (\beta)+\cos (\gamma)}{\sin (\alpha) \sin (\beta)} .
\end{gathered}
$$

Note that the first two relations have Euclidean counterparts (obtained by letting $\kappa \rightarrow 0$ ), but not the third one which is specific to nonzero curvatures.

\section{Appendix B: Periodic boundary conditions on the hyperbolic plane}

Generically, implementing periodic boundary conditions consists in choosing a primitive cell containing the physical system such that it can be infinitely replicated to tile the whole space. So, prior to building periodic boundary conditions, one needs to know the allowed tilings of the space under consideration. Here, for simplicity, we will limit ourself to regular cells and so to regular tilings.

On the hyperbolic plane $H_{2}$, an infinity of regular tilings $\{p, q\}$ are allowed if $p$ (the number of edges of the primitive cell) and $q$ (the number of cells meeting at each vertex of the tiling) verify the following condition

$$
(p-2)(q-2)>4 .
$$

This, therefore, opens the possibility to have an infinite number of possible periodic boundary conditions.

To ensure smoothness and consistency, the edges of the primitive cell of any periodic boundary condition have to be paired in a specific way: leaving the cell through one edge implies to come back by another edge, a process which should be equivalent to exploring the whole tiling of the plane. Constraints thus arise on how cell edges are paired together in addition to those on the shape of the cell. We give in the following a rapid overview of how to classify and construct periodic boundary conditions in $H^{2}$ by describing cell shapes and edge pairings.

First, a fundamental polygon (primitive cell with properly paired edges) encodes all the needed information to build periodic boundary conditions and to replicate the system in 


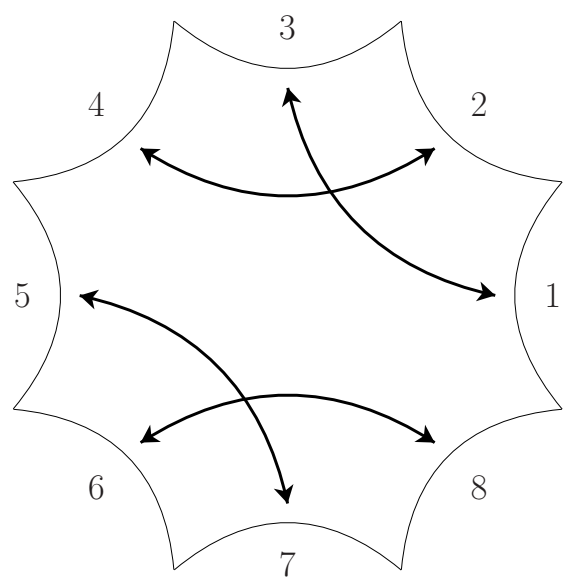

FIG. 13: Simplest fundamental polygon on the hyperbolic plane. It is associated to the $\{8,8\}$ tiling. The arrows indicate the way edges are paired.

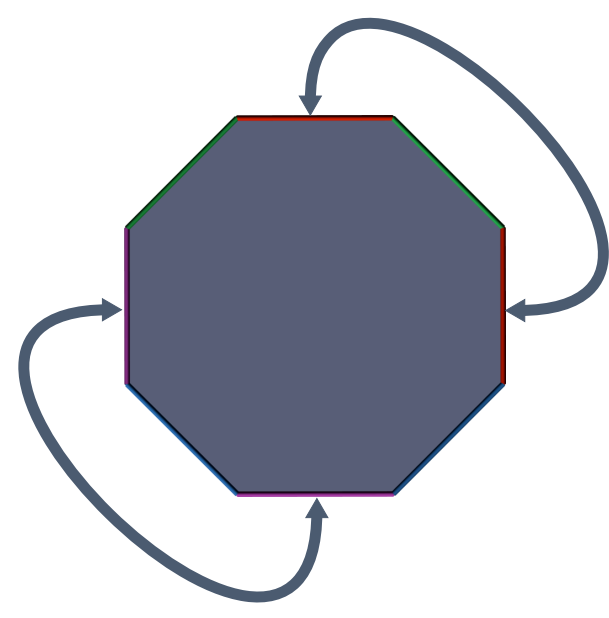

(a)

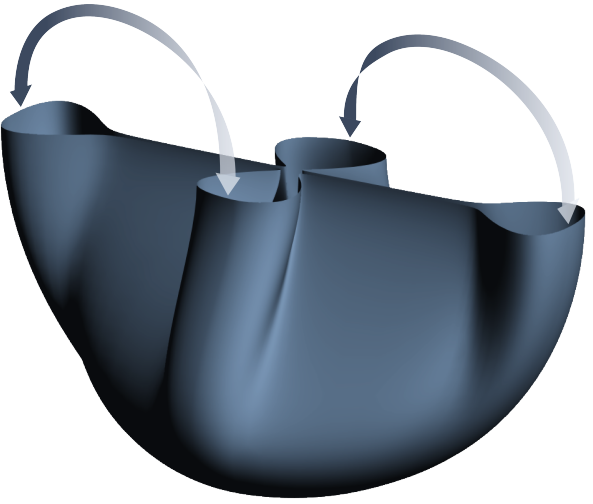

(b)

FIG. 14: Schematic representation of the "compactification" of the fundamental polygon shown in Fig. 13. The paired edges are glued together: 1 with 3 and 5 with 7 in (a); 2 with 4 and 6 with 8 in (b). The final compact manifold is a two-hole torus represented in

Fig. 15.

the entire space. The simplest (and smallest) fundamental polygon in $H^{2}$ is an octagon, corresponding to an $\{8,8\}$ tiling, with the edge pairing shown in Fig. 13 . By gluing the paired edges together (see Fig. 14 for of visualization of intermediate states) it is possible to represent the fundamental polygon as a compact manifold, also corresponding to the 


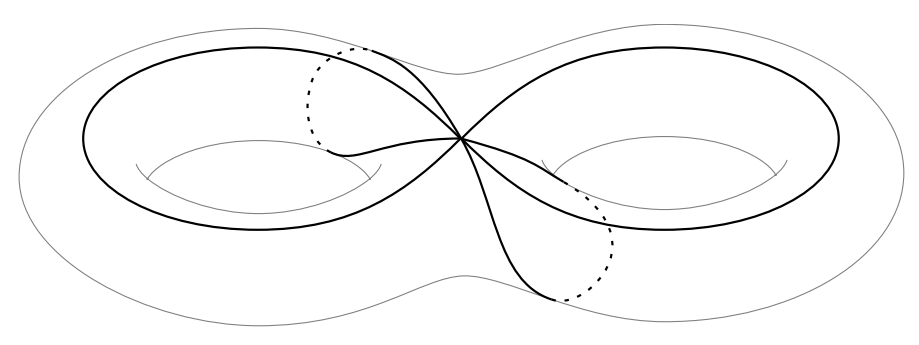

FIG. 15: Compact manifold and graph obtained by gluing the edges of the fundamental polygons of Fig. 13 .

"quotient space" (see Fig. 15). The above octagonal periodic boundary condition leads to a 2-hole torus, whose genus (number of holes, here $g=2$ ) fixes the area of the fundamental polygon through the Gauss-Bonnet theorem (see Eq. (7.4): $A=4 \pi \kappa^{-2}(g-1)$ ). As the genus is an integer, compact manifolds embedded in the hyperbolic plane can only lead to a discrete set of areas. In more technical words, an homothety cannot be applied to hyperbolic manifolds without changing the curvature. Here, the octagonal fundamental polygon cannot be scaled at constant curvature to allow one to study a bigger system for instance. Therefore, to change the area of the fundamental polygon in $H^{2}$, one as to vary its genus, which in turn implies to change its symmetry, more particularly its number of edges.

To classify all the possibilities (for regular polygons), one can use the properties of the graph formed by glued edges of the fundamental polygon and embedded in the associated $g$-hole torus (see Fig. 15 for an example on the 2-hole torus). The constraints on such graphs to obtain relevant periodic boundary conditions are detailed in 34 which allows one to classify and build all possible regular periodic boundary conditions in the hyperbolic plane [120]. The classification exhibits "families" that comprise graphs with a given number of vertices and a given pairing pattern but different values of the genus $g$ and that share similarities when varying $g$. In this framework, the octagonal fundamental polygon shown in Fig. 13 can be seen as the direct generalization of the square periodic boundary condition encountered in the Euclidean plane (both are in the same "family").

[1] J.-L. Barrat and J.-P. Hansen, Basic Concepts for Simple and Complex Liquids (Cambridge University Press, Cambridge, 2003). 
[2] M. J. Bowick and A. Travesset, Phys. Rep. 344, 255 (2001).

[3] D. R. Nelson, T. Piram, and S. Weinberg, Statistical Mechanics of Membranes and Surfaces (World Scientific, Singapore, 2004).

[4] K. W. Kratky, J. Comput. Phys. 37, 205 (1980).

[5] J. M. Caillol and D. Levesque, J. Chem. Phys. 94, 597 (1991).

[6] J. P. Hansen, D. Levesque, and J. J. Weis, Phys. Rev. Lett. 43, 979 (1979).

[7] J.-F. Sadoc and R. Mosseri, Geometrical frustration (Cambridge University Press, Cambridge, 1999).

[8] F. C. Frank, Proc. R. Soc. London Ser. A 215, 43 (1952).

[9] D. R. Nelson, Defects and geometry in condensed matter physics (Cambridge University Press, Cambridge, 2002).

[10] M. Kléman and J.-F. Sadoc, J. Physique Lett. 40, 569 (1979).

[11] M. Rubinstein and D. R. Nelson, Phys. Rev. B 28, 6377 (1983).

[12] D. R. Nelson, Phys. Rev. B 28, 5515 (1983).

[13] A. J. Post and E. D. Glandt, J. Chem. Phys. 85, 7349 (1986).

[14] B. P. Binks, Curr. Opinion Colloid Interface Sci. 7, 21 (2002).

[15] R. Aveyard, B. P. Binks, and J. H. Clint, Adv. Colloid Interface Sci. 100-102, 503 (2003).

[16] S. U. J. Pickering, J. Chem. Soc., Trans. 91, 2001 (1907,).

[17] P. Lipowsky et al., Nat. Mater. 4, 407 (2005).

[18] A. R. Bausch, M. J. Bowick, A. Cacciuto, A. D. Dinsmore, M. F. Hsu, D. R. Nelson, M. G. Nikolaides, A. Travesset, and D. A. Weitz, Science 299, 1716 (2003).

[19] A. B. Subramaniam, M. Abkarian, and H. A. Stone, Nat. Mater. 4, 553 (2005).

[20] A. B. Subramaniam, M. Abkarian, L. Mahadevan, and H. A. Stone, Langmuir 22, 10204 (2006).

[21] S. Tarimala, S. R. Ranabothu, J. P. Vernetti, and L. L. Dai, Langmuir 20, 5171 (2004).

[22] J.-F. Sadoc and J. Charvolin, Acta Cryst. A45, 10 (1988).

[23] J. Meunier, D. Langevin, and N. Boccara, Physics of amphiphilic layers (Springer-Verlag (Berlin, New York), 1987).

[24] D. A. Hajduk, P. E. Harper, S. M. Gruner, C. C. Honeker, G. Kim, E. L. Thomas, and L. J. Fetters, Macromolecules 27, 4063 (1994).

[25] M. Abkarian, A. B. Subramaniam, S.-H. Kim, R. J. Larsen, S.-M. Yang, and H. A. Stone, 
Phys. Rev. Lett. 99, 188301 (2007).

[26] A. B. Subramaniam, M. Abkarian, L. Mahadevan, and H. A. Stone, Nature 438, 930 (2005).

[27] M. J. Bowick and L. Giomi, Adv. Phys. 58, 449 (2009).

[28] T. L. Hill, Thermodynamics of Small Systems, Vol. 1 and 2 (Benjamin, New-York, 1963-1964).

[29] D. Hilbert and S. Cohn-Vossen, Geometry and the imagination (Chelsea Pub. Co., New York, 1952).

[30] H. S. M. Coxeter, Introduction to geometry, 2nd ed. (Wiley, New York, 1969).

[31] C. D. Modes and R. D. Kamien, Phys. Rev. E 77, 041125 (2008).

[32] C. D. Modes and R. D. Kamien, Phys. Rev. Lett. 99, 235701 (2007).

[33] J. H. P. McKean and I. M. Singer, J. Differential Geom. 1, 43 (1967).

[34] F. Sausset and G. Tarjus, J. Phys. A: Math. Gen. 40, 12873 (2007).

[35] C. Domb, Advances in Physics 9, 245 (1960).

[36] M. Mézard and G. Parisi, Eur. Phys. J. B 20, 217 (2001).

[37] A. Terras, Harmonic analysis on symmeric spaces and applications (Springer-Verlag, New York, 1985).

[38] A. Goetz, Introduction to Differential Geometry (Addison-Wesley, Reading, 1970).

[39] J.-P. Hansen and I. R. McDonald, Theory of simple liquids (Academic Press, 1986).

[40] K. Kratky and W. Schreiner, J. Comput. Phys. 47, 313 (1982).

[41] L. A. Fanti and E. D. Glandt, Molecular Simulation 2, 163 (1989).

[42] W. Schreiner and K. W. Kratky, Mol. Phys. 50, 435 (1983).

[43] J. S. Rowlinson and B. Widom, Molecular Theory of Capillarity (Clarendon, Oxford, 1982).

[44] F. Sausset, G. Tarjus, and P. Viot, J. Stat. Mech. 2009, P04022 (25pp) (2009).

[45] R. Fantoni, B. Jancovici, and G. Téllez, J. Stat. Phys. 112, 27 (2003).

[46] S. Lishchuk, Physica A 369, 266 (2006).

[47] S. Helgason, Indagationes Mathematicae 16, 531 (2005).

[48] B. J. Gonzalez and E. R. Negrin, PNAS 125, 3243 (1997).

[49] A. J. Post and E. D. Glandt, J. Chem. Phys. 88, 5805 (1988).

[50] A. D. Buckingham and B. D. Utting, Annu. Rev. Phys. Chem. 21, 287 (1970).

[51] B. Jancovici and G. Téllez, J. Stat. Phys. 91, 953 (1998).

[52] J. M. Caillol, J. Chem. Phys. 96, 1455 (1992).

[53] J. M. Caillol and D. Levesque, J. Chem. Phys. 96, 1477 (1992). 
[54] J. M. Caillol, D. Levesque, J. J. Weis, and J. P. Hansen, J. Stat. Phys. 28, 325 (1982).

[55] J. Caillol and D. Gilles, J. Stat. Phys. 100, 905 (2000).

[56] J.-M. Caillol, J. Chem. Phys. 99, 8953 (1993).

[57] J. P. Straley, Phys. Rev. B 30, 6592 (1984).

[58] J. P. Straley, Phys. Rev. B 34, 405 (1986).

[59] J. Tobochnik and P. M. Chapin, J. Chem. Phys. 88, 5824 (1988).

[60] S. P. Giarritta, M. Ferrario, and P. Giaquinta, Physica A 187, 456 (1992).

[61] S. Giarritta, M. Ferrario, and P. Giaquinta, Physica A 201, 649 (1993).

[62] J. M. Caillol, J. Chem. Phys. 109, 4885 (1998).

[63] J. M. Caillol and D. Levesque, Phys. Rev. B 33, 499 (1986).

[64] J. M. Caillol, J. Chem. Phys. 111, 6528 (1999).

[65] A. Pérez-Garrido and M. A. Moore, Phys. Rev. B 58, 9677 (1998).

[66] M. W. Roth and M. K. Balasubramanya, Phys. Rev. B 62, 17043 (2000).

[67] M. A. Moore and A. Pérez-Garrido, Phys. Rev. Lett. 82, 4078 (1999).

[68] F. Sausset, G. Tarjus, and P. Viot, Phys. Rev. Lett. 101, 155701 (2008).

[69] F. Sausset and G. Tarjus, Phys. Rev. Lett. 104, 065701 (2010).

[70] F. Sausset, G. Tarjus, and D. R. Nelson, Phys. Rev. E 81, 031504 (2010).

[71] W. Schreiner and K. Kratky, J. Chem. Soc., Trans. 2 78, 379 (1982).

[72] L. T. Wille, J. Phys. A: Math. Gen. 20, L1211 (1987).

[73] J.-M. Caillol, J. Phys. A: Math. Gen. 37, 3077 (2004).

[74] C. Monthus and C. Texier, J. Phys. A: Math. Gen. 29, 2399 (1996).

[75] J. Nissfolk, T. Ekholm, and C. Elvingson, J. Chem. Phys. 119, 6423 (2003).

[76] P. Castro-Villarreal, arXiv:1005.0650.

[77] F. Sausset and G. Tarjus, Philos. Mag. 88, 4025 (2008).

[78] S. V. Lishchuk, Europhys. Lett. 85, 56001 (2009).

[79] M. L. de Haro, A. Santos, and S. B. Yuste, J. Chem. Phys. 129, 116101 (2008).

[80] D. R. Nelson and B. I. Halperin, Phys. Rev. B 19, 2457 (1979).

[81] A. P. Young, Phys. Rev. B 19, 1855 (1979).

[82] J. P. Hansen and P. Viot, J. Stat. Phys. 38, 823 (1985).

[83] F. Cornu and B. Jancovici, J. Stat. Phys. 49, 33 (1987).

[84] G. Orkoulas and A. Z. Panagiotopoulos, J. Chem. Phys. 104, 7205 (1996). 
[85] P. Choquard, B. Piller, and R. Rentsch, J. Stat. Phys. 46, 599 (1987).

[86] P. A. Martin, Rev. Mod. Phys. 60, 1075 (1988).

[87] B. Jancovici and G. Téllez, J. Stat. Phys. 116, 205 (2004).

[88] M. Baus and J.-P. Hansen, Phys. Rep. 59, 1 (1980).

[89] B. Jancovici, Phys. Rev. Lett. 46, 386 (1981).

[90] J. Caillol, J. Physique Lett. 42, 245 (1981).

[91] M. Hastings, J. Stat. Phys. 90, 311 (1998).

[92] R. Fantoni and G. Tellez, J. Stat. Phys. 133, 449 (2008).

[93] C. G. Callan and F. Wilczek, Nucl. Phys. B 340, 366 (1990).

[94] R. Rietman, B. Nienhuis, and J. Oitmaa, J. Phys. A: Math. Gen. 25, 6577 (1992).

[95] J. C. Anglès d'Auriac, R. Mélin, P. Chandra, and B. Douçot, J. Phys. A: Math. Gen. 34, 675 (2001).

[96] B. Doyon, J. Phys. A: Math. Gen. 37, 359 (2004).

[97] R. R. Singh, K. S. Pitzer, J. J. de Pablo, and J. M. Prausnitz, J. Chem. Phys. 92, 5463 (1990).

[98] J. D. Bernal, Nature 183, 141 (1959).

[99] J. P. Sethna, Phys. Rev. B 31, 6278 (1985).

[100] D. Kivelson, S. Kivelson, X. Zhao, Z. Nussinov, and G. Tarjus, Physica A 219, 27 (1995).

[101] G. Tarjus, S. A. Kivelson, Z. Nussinov, and P. Viot, J. Phys.: Condens. Matter 17, R1143 (2005).

[102] J. C. Maxwell, Philos. Mag. 27, 598 (1864).

[103] S. Alexander, Phys. Rep. 296, 65 (1998).

[104] M. Wyart, L. E. Silbert, S. R. Nagel, and T. A. Witten, Phys. Rev. E 72, 051306 (2005).

[105] A. J. Liu and S. R. Nagel, Nature 396, 21 (1998).

[106] P. J. Steinhardt, D. R. Nelson, and M. Ronchetti, Phys. Rev. B 28, 784 (1983).

[107] G. Leibon and D. Letscher, in Symposium on Computational Geometry (2000) pp. 341-349.

[108] We recall the reader that the terms 'ordered phase' and 'ordering transition' for finite systems, as found in spherical geometries, should be taken with a grain of salt. It is possible that, viewed in configurational space, the topography of the energy 'landscape' changes at low energies [121, but it remains true that the energy barriers involved in equilibrating the system are finite, so that the system cannot freeze in a restricted set of configurations at nonzero 
temperature. Of course, just like in glass formation, the equilibration time may be extremely large, which in practice leads to freezing phenomena. In hyperbolic geometry, for which the system may be of infinite extent, thermodynamic phase transitions are on the other hand well defined (see Sec. IV and V).

[109] J. J. Thomson, Philos. Mag. 7, 237 (1904).

[110] D. Caspar and A. Klug, Cold Spring Harb. Symp. Quant. Biol., 27, 1 (1962).

[111] A. Travesset, Phys. Rev. E 72, 036110 (2005).

[112] M. J. Bowick, D. R. Nelson, and A. Travesset, Phys. Rev. B 62, 8738 (2000).

[113] M. Bowick, H. Shin, and A. Travesset, Phys. Rev. E 75, 021404 (2007).

[114] V. Vitelli, J. B. Lucks, and D. R. Nelson, PNAS 103, 12323 (2006).

[115] D. Nelson and L. Peliti, J. Phys. France 48, 1085 (1987).

[116] M. Bowick, A. Cacciuto, D. R. Nelson, and A. Travesset, Phys. Rev. Lett. 89, 185502 (2002).

[117] R. Backofen, A. Voigt, and T. Witkowski, Phys. Rev. E 81, 025701 (2010).

[118] M. J. W. Dodgson and M. A. Moore, Phys. Rev. B 55, 3816 (1997).

[119] T. Einert, P. Lipowsky, J. Schilling, M. J. Bowick, and A. R. Bausch, Langmuir 21, 12076 $(2005)$

[120] A tool to build such periodic boundary conditions can be found at the following address: http://physics.technion.ac.il/ sausset/CLP.html.

[121] D. H. E. Gross, Phys. Rep. 279, 119 (1997). 